\title{
MEASUREMENT AND INTERPRETATION OF ELECTROKINETIC PHENOMENA
}

\section{(IUPAC Technical Report)}

\author{
Prepared for publication by \\ A. V. DELGADO ${ }^{1,+}$, F. GONZÁLEZ-CABALLERO ${ }^{1}$, R. J. HUNTER ${ }^{2}$, L. K. KOOPAL ${ }^{3}$, AND \\ J. LYKLEMA ${ }^{3}$ \\ ${ }^{1}$ University of Granada, Granada, Spain; ${ }^{2}$ University of Sydney, Sydney, Australia; ${ }^{3}$ Wageningen \\ University, Wageningen, The Netherlands
}

With contributions from (in alphabetical order): S. Alkafeef, College of Technological Studies, Hadyia, Kuwait; E. Chibowski, Maria Curie Sklodowska University, Lublin, Poland; C. Grosse, Universidad Nacional de Tucumán, Tucumán, Argentina; A. S. Dukhin, Dispersion Technology, Inc., New York, USA; S. S. Dukhin, Institute of Water Chemistry, National Academy of Science, Kiev, Ukraine; K. Furusawa, University of Tsukuba, Tsukuba, Japan; R. Jack, Malvern Instruments, Ltd., Worcestershire, UK; N. Kallay, University of Zagreb, Zagreb, Croatia; M. Kaszuba, Malvern Instruments, Ltd., Worcestershire, UK; M. Kosmulski, Technical University of Lublin, Lublin, Poland; R. Nöremberg, BASF AG, Ludwigshafen, Germany; R. W. O’Brien, Colloidal Dynamics, Inc., Sydney, Australia; V. Ribitsch, University of Graz, Graz, Austria; V. N. Shilov, Institute of Biocolloid Chemistry, National Academy of Science, Kiev, Ukraine; F. Simon, Institut für Polymerforschung, Dresden, Germany; C. Werner; Institut für Polymerforschung, Dresden, Germany; A. Zhukov, University of St. Petersburg, Russia; R. Zimmermann, Institut für Polymerforschung, Dresden, Germany

\footnotetext{
*Membership of the Division Committee during preparation of this report (2004-2005) was as follows:

President: R. D. Weir (Canada); Vice President: C. M. A. Brett (Portugal); Secretary: M. J. Rossi (Switzerland); Titular Members: G. H. Atkinson (USA); W. Baumeister (Germany); R. Fernández-Prini (Argentina); J. G. Frey (UK); R. M. Lynden-Bell (UK); J. Maier (Germany); Z.-Q. Tian (China); Associate Members: S. Califano (Italy); S. Cabral de Menezes (Brazil); A. J. McQuillan (New Zealand); D. Platikanov (Bulgaria); C. A. Royer (France); National Representatives: J. Ralston (Australia); M. Oivanen (Finland); J. W. Park (Korea); S. Aldoshin (Russia); G. Vesnaver (Slovenia); E. L. J. Breet (South Africa).

¥Corresponding author

Republication or reproduction of this report or its storage and/or dissemination by electronic means is permitted without the need for formal IUPAC permission on condition that an acknowledgment, with full reference to the source, along with use of the copyright symbol $\odot$, the name IUPAC, and the year of publication, are prominently visible. Publication of a translation into another language is subject to the additional condition of prior approval from the relevant IUPAC National Adhering Organization.
} 


\title{
Measurement and interpretation of electrokinetic phenomena
}

\section{(IUPAC Technical Report)}

\begin{abstract}
In this report, the status quo and recent progress in electrokinetics are reviewed. Practical rules are recommended for performing electrokinetic measurements and interpreting their results in terms of well-defined quantities, the most familiar being the $\zeta$-potential or electrokinetic potential. This potential is a property of charged interfaces, and it should be independent of the technique used for its determination. However, often the $\zeta$-potential is not the only property electrokinetically characterizing the electrical state of the interfacial region; the excess conductivity of the stagnant layer is an additional parameter. The requirement to obtain the $\zeta$-potential is that electrokinetic theories be correctly used and applied within their range of validity. Basic theories and their application ranges are discussed. A thorough description of the main electrokinetic methods is given; special attention is paid to their ranges of applicability as well as to the validity of the underlying theoretical models. Electrokinetic consistency tests are proposed in order to assess the validity of the $\zeta$-potentials obtained. The recommendations given in the report apply mainly to smooth and homogeneous solid particles and plugs in aqueous systems; some attention is paid to nonaqueous media and less ideal surfaces.
\end{abstract}

Keywords: Electrokinetics; zeta potential; conductivity; aqueous systems; surface conductivity; IUPAC Physical and Biophysical Chemistry Division.

\section{CONTENTS}

1. INTRODUCTION

1.1 Electrokinetic phenomena

1.2 Definitions

1.3 Model of charges and potentials in the vicinity of a surface

1.4 Plane of shear, electrokinetic potential, and electrokinetic charge density

1.5 Basic problem: Evaluation of $\zeta$-potentials

1.6 Purpose of the document

2. ELEMENTARY THEORY OF ELECTROKINETIC PHENOMENA

3. SURFACE CONDUCTIVITY AND ELECTROKINETIC PHENOMENA

4. METHODS

\subsection{Electrophoresis}

4.1.1 Operational definitions; recommended symbols and terminology; relationship between the measured quantity and $\zeta$-potential

4.1.2 How and under which conditions the electrophoretic mobility can be converted into $\zeta$-potential

4.1.3 Experimental techniques available: Samples 
4.2 Streaming current and streaming potential

4.2.1 Operational definitions; recommended symbols and terminology; conversion of the measured quantities into $\zeta$-potential

4.2.2 Samples that can be studied

4.2.3 Sample preparation

4.3 Electro-osmosis

4.3.1 Operational definitions; recommended symbols and terminology; conversion of the measured quantities into $\zeta$-potential

4.3.2 Samples that can be studied

4.3.3 Sample preparation and standard samples

4.4 Experimental determination of surface conductivity

4.5 Dielectric dispersion

4.5.1 Operational definitions; recommended symbols and terminology; conversion of the measured quantities into $\zeta$-potential

4.5.2 Dielectric dispersion and $\zeta$-potential: Models

4.5.3 Experimental techniques available

4.5.4 Samples for LFDD measurements

4.6 Electroacoustics

4.6.1 Operational definitions; recommended symbols and terminology; experimentally available quantities

4.6.2 Estimation of the $\zeta$-potential from $U_{C V}, I_{C V}$, or $A_{E S A}$

4.6.3 Experimental procedures

4.6.4 Samples for calibration

5. ELECTROKINETICS IN NONAQUEOUS SYSTEMS

5.1 Difference with aqueous systems: Permittivity

5.2 Experimental requirements of electrokinetic techniques

5.3 Conversion of electrokinetic data into $\zeta$-potentials

6. REMARKS ON NON-IDEAL SURFACES

6.1 General comments

6.2 Hard surfaces

6.2.1 Size effects

6.2.2 Shape effects

6.2.3 Surface roughness

6.2.4 Chemical surface heterogeneity

6.3 Soft particles

6.3.1 Charged particles with a soft uncharged layer

6.3.2 Uncharged particles with a soft charged layer

6.3.3 Charged particles with a soft charged layer

6.3.4 Ion-penetrable or partially penetrable particles

6.3.5 Liquid droplets and gas bubbles in a liquid

7. DISCUSSION AND RECOMMENDATIONS

8. APPENDIX I. CALCULATION OF THE LOW-FREQUENCY DIELECTRIC DISPERSION OF SUSPENSIONS

9. ACKNOWLEDGMENTS

10. REFERENCES

11. LIST OF SYMBOLS 


\section{INTRODUCTION}

\subsection{Electrokinetic phenomena}

Electrokinetic phenomena (EKP) can be loosely defined as all those phenomena involving tangential fluid motion adjacent to a charged surface. They are manifestations of the electrical properties of interfaces under steady-state and isothermal conditions. In practice, they are often the only source of information available on those properties. For this reason, their study constitutes one of the classical branches of colloid science, electrokinetics, which has been developed in close connection with the theories of the electrical double layer (EDL) and of electrostatic surface forces [1-4].

From the point of view of nonequilibrium thermodynamics, EKP are typically cross-phenomena, because thermodynamic forces of a certain kind create fluxes of another type. For instance, in electroosmosis and electrophoresis, an electric force leads to a mechanical motion, and in streaming current (potential), an applied mechanical force produces an electric current (potential). First-order phenomena may also provide valuable information about the electrical state of the interface: for instance, an external electric field causes the appearance of a surface current, which flows along the interfacial region and is controlled by the surface conductivity of the latter. If the applied field is alternating, the electric permittivity of the system as a function of frequency will display one or more relaxations. The characteristic frequency and amplitude of these relaxations may yield additional information about the electrical state of the interface. We consider these first-order phenomena as closely related to EKP.

\subsection{Definitions}

Here follows a brief description of the main and related EKP [1-9].

- Electrophoresis is the movement of charged colloidal particles or polyelectrolytes, immersed in a liquid, under the influence of an external electric field. The electrophoretic velocity, $v_{\mathrm{e}}\left(\mathrm{m} \mathrm{s}^{-1}\right)$, is the velocity during electrophoresis. The electrophoretic mobility, $u_{\mathrm{e}}\left(\mathrm{m}^{2} \mathrm{~V}^{-1} \mathrm{~s}^{-1}\right)$, is the magnitude of the velocity divided by the magnitude of the electric field strength. The mobility is counted positive if the particles move toward lower potential (negative electrode) and negative in the opposite case.

- Electro-osmosis is the motion of a liquid through an immobilized set of particles, a porous plug, a capillary, or a membrane, in response to an applied electric field. It is the result of the force exerted by the field on the counter-charge in the liquid inside the charged capillaries, pores, etc. The moving ions drag the liquid in which they are embedded along. The electro-osmotic velocity, $v_{\mathrm{eo}}\left(\mathrm{m} \mathrm{s}^{-1}\right)$, is the uniform velocity of the liquid far from the charged interface. Usually, the measured quantity is the volume flow rate of liquid $\left(\mathrm{m}^{3} \mathrm{~s}^{-1}\right)$ through the capillary, plug, or membrane, divided by the electric field strength, $Q_{\mathrm{eo}, E}\left(\mathrm{~m}^{4} \mathrm{~V}^{-1} \mathrm{~s}^{-1}\right)$, or divided by the electric current, $Q_{\mathrm{eo}, I}\left(\mathrm{~m}^{3} \mathrm{C}^{-1}\right)$. A related concept is the electro-osmotic counter-pressure, $\Delta p_{\mathrm{eo}}(\mathrm{Pa})$, the pressure difference that must be applied across the system to stop the electro-osmotic volume flow. The value $\Delta p_{\mathrm{eo}}$ is considered to be positive if the high pressure is on the higher electric potential side.

- $\quad$ Streaming potential (difference), $U_{\text {str }}(\mathrm{V})$, is the potential difference at zero electric current, caused by the flow of liquid under a pressure gradient through a capillary, plug, diaphragm, or membrane. The difference is measured across the plug or between the ends of the capillary. Streaming potentials are created by charge accumulation caused by the flow of counter-charges inside capillaries or pores.

- $\quad$ Streaming current, $I_{\text {str }}(\mathrm{A})$, is the current through the plug when the two electrodes are relaxed and short-circuited. The streaming current density, $j_{\mathrm{str}}\left(\mathrm{A} \mathrm{m}^{-2}\right)$, is the streaming current per area.

- Dielectric dispersion is the change of the electric permittivity of a suspension of colloidal particles with the frequency of an applied alternating current (ac) field. For low and middle frequen- 
cies, this change is connected with the polarization of the ionic atmosphere. Often, only the lowfrequency dielectric dispersion (LFDD) is investigated.

- $\quad$ Sedimentation potential, $U_{\text {sed }}(\mathrm{V})$, is the potential difference sensed by two identical electrodes placed some vertical distance $L$ apart in a suspension in which particles are sedimenting under the effect of gravity. The electric field generated, $U_{\text {sed }} / L$, is known as the sedimentation field, $E_{\text {sed }}$ $\left(\mathrm{V} \mathrm{m}^{-1}\right)$. When the sedimentation is produced by a centrifugal field, the phenomenon is called centrifugation potential.

- $\quad$ Colloid vibration potential, $U_{\mathrm{CV}}(\mathrm{V})$, measures the ac potential difference generated between two identical relaxed electrodes, placed in the dispersion, if the latter is subjected to an (ultra)sonic field. When a sound wave travels through a colloidal suspension of particles whose density differs from that of the surrounding medium, inertial forces induced by the vibration of the suspension give rise to a motion of the charged particles relative to the liquid, causing an alternating electromotive force. The manifestations of this electromotive force may be measured, depending on the relation between the impedance of the suspension and that of the measuring instrument, either as $U_{\mathrm{CV}}$ or as colloid vibration current, $I_{\mathrm{CV}}(\mathrm{A})$.

- Electrokinetic sonic amplitude (ESA) method provides the amplitude, $A_{\mathrm{ESA}}(\mathrm{Pa})$, of the (ultra)sonic field created by an ac electric field in a dispersion; it is the counterpart of the colloid vibration potential method.

- $\quad$ Surface conduction is the excess electrical conduction tangential to a charged surface. It will be represented by the surface conductivity, $K^{\sigma}(\mathrm{S})$, and its magnitude with respect to the bulk conductivity is frequently accounted for by the Dukhin number, $D u$ (see eq. 12 below).

\subsection{Model of charges and potentials in the vicinity of a surface}

Charges. The electrical state of a charged surface is determined by the spatial distribution of ions around it. Such a distribution of charges has traditionally been called EDL, although it is often more complex than just two layers, and some authors have proposed the term "electrical interfacial layer". We propose here to keep the traditional terminology, which is used widely in the field. The simplest picture of the EDL is a physical model in which one layer of the EDL is envisaged as a fixed charge, the surface or titratable charge, firmly bound to the particle or solid surface, while the other layer is distributed more or less diffusely within the solution in contact with the surface. This layer contains an excess of counterions (ions opposite in sign to the fixed charge), and has a deficit of co-ions (ions of the same sign as the fixed charge.)

For most purposes, a more elaborate model is necessary [3,10]: the uncharged region between the surface and the locus of hydrated counterions is called the Stern layer, whereas ions beyond it form the diffuse layer or Gouy layer (also, Gouy-Chapman layer). In some cases, the separation of the EDL into a charge-free Stern layer and a diffuse layer is not sufficient to interpret experiments. The Stern layer is then subdivided into an inner Helmholtz layer (IHL), bounded by the surface and the inner Helmholtz plane (IHP) and an outer Helmholtz layer (OHL), located between the IHP and the outer Helmholtz plane (OHP). This situation is shown in Fig. 1 for a simple case. The necessity of this subdivision may occur when some ion types (possessing a chemical affinity for the surface in addition to purely Coulombic interactions), are specifically adsorbed on the surface, whereas other ion types interact with the surface charge only through electrostatic forces. The IHP is the locus of the former ions, and the OHP determines the beginning of the diffuse layer, which is the generic part of the EDL (i.e., the part governed by purely electrostatic forces). The fixed surface-charge density is denoted $\sigma^{0}$, the charge density at the IHP $\sigma^{\mathrm{i}}$, and that in the diffuse layer $\sigma^{\mathrm{d}}$. As the system is electroneutral

$$
\sigma^{0}+\sigma^{\mathrm{i}}+\sigma^{\mathrm{d}}=0
$$




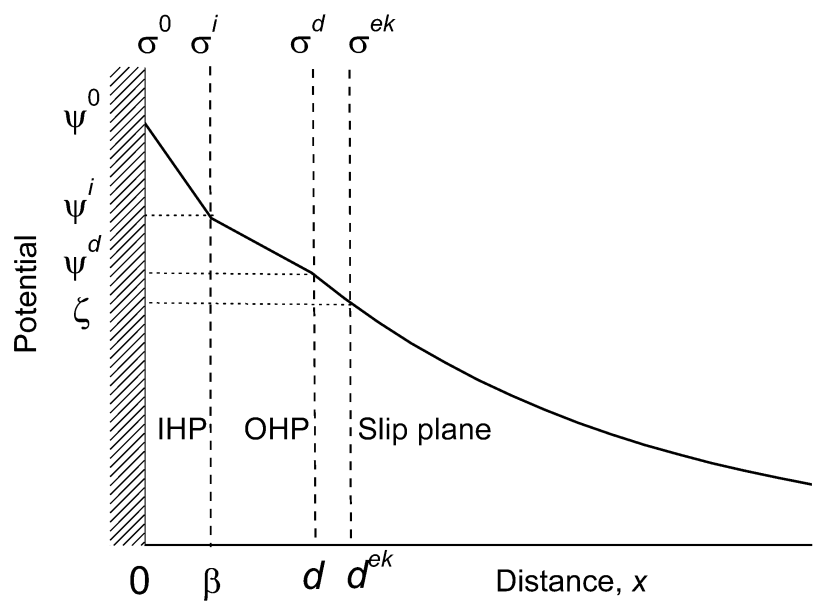

Fig. 1 Schematic representation of the charges and potentials at a positively charged interface. The region between the surface (electric potential $\psi^{0}$; charge density $\sigma^{0}$ ) and the IHP (distance $\beta$ from the surface) is free of charge. The IHP (electric potential $\psi^{\mathrm{i}}$; charge density $\sigma^{\mathrm{i}}$ ) is the locus of specifically adsorbed ions. The diffuse layer starts at $x=d(\mathrm{OHP})$, with potential $\psi^{\mathrm{d}}$ and charge density $\sigma^{\mathrm{d}}$. The slip plane or shear plane is located at $x=d^{\mathrm{ek}}$. The potential at the slip plane is the electrokinetic or zeta-potential, $\zeta$; the electrokinetic charge density is $\sigma^{\mathrm{ek}}$.

Potentials. As isolated particles cannot be linked directly to an external circuit, it is not possible to change their surface potential at will by applying an external field. Contrary to mercury and other electrodes, the surface potential, $\psi^{0}$, of a solid is therefore not capable of operational definition, meaning that it cannot be unambiguously measured without making model assumptions. As a consequence, for disperse systems it is the surface charge that is the primary parameter, rather than the surface potential. The potential at the OHP, at distance $d$ from the surface, is called the diffuse-layer potential, $\psi^{\mathrm{d}}$ (sometimes also known as Stern potential): it is the potential at the beginning of the diffuse part of the double layer. The potential at the IHP, located at distance $\beta(0 \leq \beta \leq d)$ from the surface, the IHP potential, is given the symbol $\psi^{i}$. All potentials are defined with respect to the potential in bulk solution.

Concerning the ions in the EDL, some further comments can be of interest. Usually, a distinction is made between indifferent and specifically adsorbing ions. Indifferent ions adsorb through Coulomb forces only; hence, they are repelled by surfaces of like sign, attracted by surfaces of opposite sign, and do not preferentially adsorb on an uncharged surface. Specifically adsorbing ions possess a chemical or specific affinity for the surface in addition to the Coulomb interaction, where chemical or specific is a collective adjective, embracing all interactions other than those purely Coulombic. It was recommended in [10], and is now commonly in use to restrict the notion of surface ions to those that are constituents of the solid, and hence are present on the surface, and to proton and hydroxyl ions. The former are covalently adsorbed. The latter are included because they are always present in aqueous solutions, their adsorption can be measured (e.g., by potentiometric titration) and they have, for many surfaces, a particularly high affinity. The term specifically adsorbed then applies to the sorption of all other ions having a specific affinity to the surface in addition to the generic Coulombic contribution. Specifically adsorbed charges are located within the Stern layer.

\subsection{Plane of shear, electrokinetic potential, and electrokinetic charge density}

Tangential liquid flow along a charged solid surface can be caused by an external electric field (electrophoresis, electro-osmosis) or by an applied mechanical force (streaming potential, current). Experience and recent molecular dynamic simulations [11] have shown that in such tangential motion 
usually a very thin layer of fluid adheres to the surface: it is called the hydrodynamically stagnant layer, which extends from the surface to some specified distance, $d^{\mathrm{ek}}$, where a so-called hydrodynamic slip plane is assumed to exist. For distances to the wall, $x<d^{\mathrm{ek}}$, one has the stagnant layer in which no hydrodynamic flows can develop. Thus, we can speak of a distance-dependent viscosity with roughly a step-function dependence [12]. The space charge for $x>d^{\text {ek }}$ is hydrodynamically mobile and electrokinetically active, and a particle (if spherical) behaves hydrodynamically as if it had a radius $a+d^{\mathrm{ek}}$. The space charge for $x<d^{\mathrm{ek}}$ is hydrodynamically immobile, but can still be electrically conducting. The potential at the plane where slip with respect to bulk solution is postulated to occur is identified as the electrokinetic or zeta-potential, $\zeta$. The diffuse charge at the solution side of the slip plane equals the negative of the electrokinetic (particle) charge, $\sigma^{\mathrm{ek}}$.

General experience indicates that the plane of shear is located very close to the OHP. Both planes are abstractions of reality. The OHP is interpreted as a sharp boundary between the diffuse and the nondiffuse parts of the EDL, but it is very difficult to locate it exactly. Likewise, the slip plane is interpreted as a sharp boundary between the hydrodynamically mobile and immobile fluid. In reality, none of these transitions is sharp. However, liquid motion may be hindered in the region where ions experience strong interactions with the surface. Therefore, it is feasible that the immobilization of the fluid extends further out of the surface than the beginning of the diffuse part of the EDL. This means that, in practice, the $\zeta$-potential is equal to or lower in magnitude than the diffuse-layer potential, $\psi^{\mathrm{d}}$. In the latter case, the difference between $\psi^{\mathrm{d}}$ and $\zeta$ is a function of the ionic strength: at low ionic strength, the decay of the potential as a function of distance is small and $\zeta \cong \psi^{\mathrm{d}}$; at high ionic strength, the decay is steeper and $|\zeta| \leq\left|\psi^{\mathrm{d}}\right|$. A similar reasoning applies to the electrokinetic charge, as compared to the diffuse charge.

\subsection{Basic problem: Evaluation of $\zeta$-potentials}

The notion of slip plane is generally accepted in spite of the fact that there is no unambiguous way of locating it. It is also accepted that $\zeta$ is fully defined by the nature of the surface, its charge (often determined by $\mathrm{pH}$ ), the electrolyte concentration in the solution, and the nature of the electrolyte and of the solvent. It can be said that for any interface with all these parameters fixed, $\zeta$ is a well-defined property.

Experience demonstrates that different researchers often find different $\zeta$-potentials for supposedly identical interfaces. Sometimes, the surfaces are not in fact identical: the high specific surface area and surface reactivity of colloidal systems make $\zeta$ very sensitive to even minor amounts of impurities in solution. This can partly explain variations in electrokinetic determinations from one laboratory to another. Alternatively, since $\zeta$ is not a directly measurable property, it may be that an inappropriate model has been used to convert the electrokinetic signal into a $\zeta$-potential. The level of sophistication required (for the model) depends on the situation and on the particular phenomena investigated. The choice of measuring technique and of the theory used depends to a large extent on the purpose of the electrokinetic investigation.

There are instances in which the use of simple models can be justified, even if they do not yield the correct $\zeta$-potential. For example, if electrokinetic measurements are used as a sort of quality-control tool, one is interested in rapidly (online) detecting modifications in the electrical state of the interface rather than in obtaining accurate $\zeta$-potentials. On the other hand, when the purpose is to compare the calculated values of $\zeta$ of a system under given conditions using different electrokinetic techniques, it may be essential to find a true $\zeta$-potential. The same applies to those cases in which $\zeta$ will be used to perform calculations of other physical quantities, such as the Gibbs interaction energy between particles. Furthermore, there may be situations in which the use of simple theories may be misleading even for simple quality control. For example, there are ranges of $\zeta$-potential and double-layer thickness for which the electrophoretic mobility does not depend linearly on $\zeta$, as assumed in the simple models. Two samples might have the same true $\zeta$-potential and quite different mobilities because of their different 
sizes. The simple theory would lead us to believe that their electrical surface characteristics are different when they are not.

An important complicating factor in the reliable estimation of $\zeta$ is the possibility that charges behind the plane of shear may contribute to the excess conductivity of the double layer (stagnant-layer or inner-layer conductivity.) If it is assumed that charges located between the surface and the plane of shear are electrokinetically inactive, then the $\zeta$-potential will be the only interfacial quantity explaining the observed electrokinetic signal.

Otherwise, a correct quantitative explanation of EKP will require the additional estimation of the stagnant-layer conductivity (SLC). This requires more elaborate treatments [2,3,13-17] than standard or classical theories, in which only conduction at the solution side of the plane of shear is considered.

It should be noted that there are a number of situations where electrokinetic measurements, without further interpretation, provide extremely useful and unequivocal information, of great value for technological purposes. The most important of these situations are

- identification of the isoelectric point (or point of zero $\zeta$-potential) in titrations with a potential determining ion (e.g., $\mathrm{pH}$ titration);

- identification of the isoelectric point in titrations with other ionic reagents such as surfactants or polyelectrolytes; and

- identification of a plateau in the adsorption of an ionic species indicating optimum dosage for a dispersing agent.

In these cases, the complications and digressions, which are discussed below, are essentially irrelevant. The electrokinetic property (or the estimated $\zeta$-potential) is then zero or constant and that fact alone is of value.

\subsection{Purpose of the document}

The present document is intended to deal mainly with the following issues, related to the role of the different EKP as tools for surface chemistry research. Specifically, its aims are:

- Describe and codify the main and related EKP and the quantities involved in their definitions.

- Give a general overview of the main experimental techniques that are available for electrokinetic characterization.

- $\quad$ Discuss the models for the conversion of the experimental signal into $\zeta$-potential and, where appropriate, other double-layer characteristics.

- Identify the validity range of such models, and the way in which they should be applied to any particular experimental situation.

The report first discusses the most widely used EKP and techniques, such as electrophoresis, streaming-potential, streaming current, or electro-osmosis. Attention is also paid to the rapidly growing techniques based on dielectric dispersion and electro-acoustics.

\section{ELEMENTARY THEORY OF ELECTROKINETIC PHENOMENA}

All electrokinetic effects originate from two generic phenomena, namely, the electro-osmotic flow and the convective electric surface current within the EDL. For nonconducting solids, Smoluchowski [18] derived equations for these generic phenomena, which allowed an extension of the theory to all other specific EKP. Smoluchowski's theory is valid for any shape of a particle or pores inside a solid, provided the (local) curvature radius $a$ largely exceeds the Debye length $\kappa^{-1}$ :

$$
\kappa a \gg 1
$$

where $\kappa$ is defined as 


$$
\kappa=\left\{\frac{\sum_{i=1}^{N} e^{2} z_{i}^{2} n_{i}}{\varepsilon_{\mathrm{rs}} \varepsilon_{0} k T}\right\}^{1 / 2}
$$

with $e$ the elementary charge, $z_{i}, n_{i}$ the charge number and number concentration of ion $i$ (the solution contains $N$ ionic species), $\varepsilon_{\mathrm{rs}}$ the relative permittivity of the electrolyte solution, $\varepsilon_{0}$ the electric permittivity of vacuum, $k$ the Boltzmann constant, and $T$ the thermodynamic temperature. Note that under condition (2), a curved surface can be considered as flat for any small section of the double layer. This condition is traditionally called the "thin double-layer approximation", but we do not recommend this language, and we rather refer to this as the "large $\kappa a$ limit". Many aqueous dispersions satisfy this condition, but not those for very small particles in low ionic strength media.

Electro-osmotic flow is the liquid flow along any section of the double layer under the action of the tangential component $E_{\mathrm{t}}$ of an external field $\boldsymbol{E}$. In Smoluchowski's theory, this field is considered to be independent of the presence of the double layer, i.e., the distortion of the latter is ignored*. Also, because the EDL is assumed to be very thin compared to the particle radius, the hydrodynamic and electric field lines are parallel for large $\kappa a$. Under these conditions, it can be shown [3] that at a large distance from the surface the liquid velocity (electro-osmotic velocity), $v_{\mathrm{eo}}$, is given by

$$
v_{\mathrm{eo}}=-\frac{\varepsilon_{\mathrm{rs}} \varepsilon_{0} \zeta}{\eta} \boldsymbol{E}
$$

where $\eta$ is the dynamic viscosity of the liquid. This is the Smoluchowski equation for the electro-osmotic slip velocity. From this, the electro-osmotic flow rate of liquid per current, $Q_{\mathrm{eo}, I}\left(\mathrm{~m}^{3} \mathrm{~s}^{-1} \mathrm{~A}^{-1}\right)$, can be derived

$$
Q_{\mathrm{eo}, I}=\frac{Q_{\mathrm{eo}}}{I}=-\frac{\varepsilon_{\mathrm{rs}} \varepsilon_{0} \zeta}{\eta K_{\mathrm{L}}}
$$

$K_{\mathrm{L}}$ being the bulk liquid conductivity $\left(\mathrm{S} \mathrm{m}^{-1}\right)$ and $I$ the electric current (A).

It is impossible to quantify the distribution of the electric field and the velocity in pores with unknown or complex geometry. However, this fundamental difficulty is avoided for $\kappa a>>1$, when eqs. 4 and 5 are valid [3].

Electrophoresis is the counterpart of electro-osmosis. In the latter, the liquid moves with respect to a solid body when an electric field is applied, whereas during electrophoresis the liquid as a whole is at rest, while the particle moves with respect to the liquid under the influence of the electric field. In both phenomena, such influence on the double layer controls the relative motions of the liquid and the solid body. Hence, the results obtained in considering electro-osmosis can be readily applied for obtaining the corresponding formula for electrophoresis. The expression for the electrophoretic velocity, that is, the velocity of the particle with respect to a medium at rest, becomes, after changing the sign in eq. 4

$$
v_{\mathrm{e}}=\frac{\varepsilon_{\mathrm{rs}} \varepsilon_{0} \zeta}{\eta} \boldsymbol{E}
$$

and the electrophoretic mobility, $u_{\mathrm{e}}$

\footnotetext{
*The approximation that the structure of the double layer is not affected by the applied field is one of the most restrictive assumptions of the elementary theory of EKP.
} 


$$
u_{\mathrm{e}}=\frac{\varepsilon_{\mathrm{rs}} \varepsilon_{0} \zeta}{\eta}
$$

This equation is known as the Helmholtz-Smoluchowski (HS) equation for electrophoresis.

Let us consider a capillary with circular cross-section of radius $a$ and length $L$ with charged walls. A pressure difference between the two ends of the capillary, $\Delta p$, is produced externally to drive the liquid through the capillary. Since the fluid near the interface carries an excess of charge equal to $\sigma^{\mathrm{ek}}$, its motion will produce an electric current known as streaming current, $I_{\text {str: }}$ :

$$
I_{\text {str }}=-\frac{\varepsilon_{\mathrm{rs}} \varepsilon_{0} a^{2}}{\eta} \frac{\Delta p}{L} \zeta
$$

The observation of this current is only possible if the extremes of the capillary are connected through a low-resistance external circuit (short-circuit conditions). If this resistance is high (open circuit), transport of ions by this current leads to the accumulation of charges of opposite signs between the two ends of the capillary and, consequently, to the appearance of a potential difference across the length of the capillary, the streaming-potential, $U_{\mathrm{str}}$. This gives rise to a conduction current, $I_{\mathrm{c}}$ :

$$
I_{\mathrm{c}}=K_{\mathrm{L}} a^{2} \frac{U_{\mathrm{str}}}{L}
$$

The value of the streaming-potential is obtained by the condition of equality of the conduction and streaming currents (the net current vanishes)

$$
\frac{U_{\mathrm{str}}}{\Delta p}=\frac{\varepsilon_{\mathrm{rs}} \varepsilon_{0} \zeta}{\eta K_{\mathrm{L}}}
$$

For large $\kappa a$, eq. 10 is also valid for porous bodies.

As described, the theory is incomplete in mainly three aspects: (i) it does not include the treatment of strongly curved surfaces (i.e., surfaces for which the condition $\kappa a \gg 1$ does not apply); (ii) it neglects the effect of surface conduction both in the diffuse and the inner part of the EDL; and (iii) it neglects EDL polarization. Concerning the first point, the theoretical analysis described above is based on the assumption that the interface is flat or that its radius of curvature at any point is much larger than the double-layer thickness. When this condition is not fulfilled, the Smoluchowski theory ceases to be valid, no matter the existence or not of surface conduction of any kind. However, theoretical treatments have been devised to deal with these surface curvature effects. Roughly, in order to check if such corrections are needed, one should simply calculate the product $\kappa a$, where $a$ is a characteristic radius of curvature (e.g., particle radius, pore or capillary radius). When describing the methods below, we will give details about analytical or numerical procedures that can be used to account for this effect.

With respect to surface conductivity, a detailed account is given in Section 3, and mention will be made to it where necessary in the description of the methods. Here, it may suffice to say that it may be important when the $\zeta$-potential is moderately large ( $>50 \mathrm{mV}$, say.)

Finally, the polarization of the double layer implies accumulation of excess charge on one side of the colloidal particle and depletion on the other. The resulting induced dipole is the source of an electric field distribution that is superimposed on the applied field and affects the relative solid/liquid motion. The extent of polarization depends on surface conductivity, and its role in electrokinetics will be discussed together with the methodologies. 


\section{SURFACE CONDUCTIVITY AND ELECTROKINETIC PHENOMENA}

Surface conduction is the name given to the excess electric conduction that takes place in dispersed systems owing to the presence of the electric double layers. Excess charges in them may move under the influence of electric fields applied tangentially to the surface. The phenomenon is quantified in terms of the surface conductivity, $K^{\sigma}$, which is the surface equivalent to the bulk conductivity, $K_{\mathrm{L}} \cdot K^{\sigma}$ is a surface excess quantity just as the surface concentration $\Gamma_{i}$ of a certain species $i$. Whatever the charge distribution, $K^{\sigma}$ can always be defined through the two-dimensional analog of Ohm's law

$$
j^{\sigma}=K^{\sigma} \boldsymbol{E}
$$

where $j^{\sigma}$ is the (excess) surface current density $\left(\mathrm{A} \mathrm{m}^{-1}\right)$.

A measure of the relative importance of surface conductivity is given by the dimensionless Dukhin number, $D u$, relating surface $\left(K^{\sigma}\right)$ and bulk $\left(K_{\mathrm{L}}\right)$ conductivities

$$
D u=\frac{K^{\sigma}}{K_{\mathrm{L}} a}
$$

where $a$ is the local curvature radius of the surface. For a colloidal system, the total conductivity, $K$, can be expressed as the sum of a solution contribution and a surface contribution. For instance, for a cylindrical capillary, the following expression results:

$$
K=\left(K_{\mathrm{L}}+2 K^{\sigma} / a\right)=K_{\mathrm{L}}(1+2 D u)
$$

The factor 2 in eq. 13 applies for cylindrical geometry. For other geometries, its value may be different.

As mentioned, HS theory does not consider surface conduction, and only the solution conductivity, $K_{\mathrm{L}}$, is taken into account to derive the tangential electric field within the double layer. Thus, in addition to eq. 2, the applicability of the HS theory requires

$$
D u<<1
$$

The surface conductivity can have contributions owing to the diffuse-layer charge outside the plane of shear, $K^{\sigma \mathrm{d}}$, and to the stagnant layer $K^{\sigma \mathrm{i}}$ :

$$
K^{\sigma}=K^{\sigma \mathrm{d}}+K^{\sigma \mathrm{i}}
$$

Accordingly, $D u$ can be written as

$$
D u=\frac{K^{\sigma \mathrm{d}}}{K_{\mathrm{L}} a}+\frac{K^{\sigma \mathrm{i}}}{K_{\mathrm{L}} a}=D u^{\mathrm{d}}+D u^{\mathrm{i}}
$$

The $K^{\sigma \mathrm{d}}$ contribution is called the Bikerman surface conductivity after Bikerman, who found a simple equation for $K^{\sigma d}$ (see below). The SLC may include a contribution due to the specifically adsorbed charge and another one due to the part of the diffuse-layer charge that may reside behind the plane of shear. The charge on the solid surface is generally assumed to be immobile; it does not contribute to $K^{\sigma}$.

The conductivity in the diffuse double layer outside the plane of shear, $K^{\sigma \mathrm{d}}$, consists of two parts: a migration contribution, caused by the movement of charges with respect to the liquid; and a convective contribution, due to the electro-osmotic liquid flow beyond the shear plane, which gives rise to an additional mobility of the charges and hence leads to an extra contribution to $K^{\sigma \mathrm{d}}$. For the calculation of $K^{\sigma d}$, the Bikerman equation (eq. 17, see below) can be used. This equation expresses $K^{\sigma d}$ as a func- 
tion of the electrolyte and double-layer parameters. For a symmetrical $z-z$ electrolyte, a convenient expression is

$$
K^{\sigma \mathrm{d}}=\frac{2 e^{2} N_{\mathrm{A}} z^{2} c}{k T \kappa}\left[D_{+}\left(e^{-z e \zeta / 2 k T}-1\right)\left(1+\frac{3 m_{+}}{z^{2}}\right)+D_{-}\left(e^{z e \zeta / 2 k T}-1\right)\left(1+\frac{3 m_{-}}{z^{2}}\right)\right]
$$

where $c$ is the electrolyte amount concentration $\left(\mathrm{mol} \mathrm{m}{ }^{-3}\right), N_{\mathrm{A}}$ is the Avogadro constant $\left(\mathrm{mol}^{-1}\right)$, and $m_{+}\left(m_{-}\right)$is the dimensionless mobility of the cations (anions)

$$
m_{ \pm}=\frac{2}{3}\left(\frac{k T}{e}\right)^{2} \frac{\varepsilon_{\mathrm{rs}} \varepsilon_{0}}{\eta D_{ \pm}}
$$

where $D_{ \pm}\left(\mathrm{m}^{2} \mathrm{~s}^{-1}\right)$ are the ionic diffusion coefficients. The parameters $m_{ \pm}$indicate the relative contribution of electro-osmosis to the surface conductivity.

The extent to which $K^{\sigma}$ influences the electrokinetic behavior of the systems depends on the value of $D u$. For the Bikerman part of the conductivity, $D u^{\mathrm{d}}$ can be written explicitly. For a symmetrical $z-z$ electrolyte and identical cation and anion diffusion coefficients so that $m_{+}=m_{-}=m$ :

$$
D u^{\mathrm{d}} \equiv \frac{K^{\sigma \mathrm{d}}}{K_{\mathrm{L}} a}=\frac{2}{\kappa a}\left(1+\frac{3 m}{z^{2}}\right)\left[\cosh \left(\frac{z e \zeta}{2 k T}\right)-1\right]
$$

From this equation, it follows that $D u^{\mathrm{d}}$ is small if $\kappa a>>1$, and $\zeta$ is small. Substitution of this expression for $D u^{\mathrm{d}}$ in eq. 16 yields

$$
D u=\frac{2}{\kappa a}\left(1+\frac{3 m}{z^{2}}\right)\left[\cosh \left(\frac{z e \zeta}{2 k T}\right)-1\right]\left(1+\frac{K^{\sigma \mathrm{i}}}{K^{\sigma \mathrm{d}}}\right)
$$

This equation shows that, in general, $D u$ is dependent on the $\zeta$-potential, the ion mobility in bulk solution, and $K^{\sigma \mathrm{i}} / K^{\sigma \mathrm{d}}$. Now, the condition $D u<<1$ required for application of the HS theory is achieved for $\kappa a \gg>1$, rather low values of $\zeta$, and $K^{\sigma \mathrm{i} /} / K^{\sigma \mathrm{d}}<1$.

\section{METHODS}

\subsection{Electrophoresis}

4.1.1 Operational definitions; recommended symbols and terminology; relationship between the measured quantity and $\zeta$-potential

Electrophoresis is the translation of a colloidal particle or polyelectrolyte, immersed in a liquid, under the action of an externally applied field, $\boldsymbol{E}$, constant in time and position-independent. For uniform and not very strong electric fields, a linear relationship exists between the steady-state electrophoretic velocity, $v_{\mathrm{e}}$ (attained by the particle roughly a few milliseconds after application of the field) and the applied field

$$
v_{\mathrm{e}}=u_{\mathrm{e}} \boldsymbol{E}
$$

where $u_{\mathrm{e}}$ is the quantity of interest, the electrophoretic mobility. 


\subsubsection{How and under which conditions the electrophoretic mobility can be converted into $\zeta$-potential}

As discussed above, it is not always possible to rigorously obtain the $\zeta$-potential from measurements of electrophoretic mobility only. We give here some guidelines to check whether the system under study can be described with the standard electrokinetic models:

a. Calculate $\kappa a$ for the suspension.

b. If $\kappa a \gg>1(\kappa a>20$, say), we are in the large $\kappa a$ regime, and simple analytical models are available.

b.1 Obtain the mobility $u_{\mathrm{e}}$ for a range of indifferent electrolyte concentrations. If $u_{\mathrm{e}}$ decreases with increasing electrolyte concentration, use the HS formula, eq. 7, to obtain $\zeta$.

b.1.1 If the $\zeta$ value obtained is low $(\zeta \leq 50 \mathrm{mV}$, say), concentration polarization is negligible, and one can trust the value of $\zeta$.

b.1.2 If $\zeta$ is rather high ( $\zeta>50 \mathrm{mV}$, say), then HS theory is not applicable. One has to use more elaborate models. The possibilities are: (i) the numerical calculations of O'Brien and White [22]; (ii) the equation derived by Dukhin and Semenikhin [5] for symmetrical $z-z$ electrolytes

$$
\begin{aligned}
& \frac{3}{2} \frac{\eta e}{\varepsilon_{\mathrm{rs}} \varepsilon_{0} k T} u_{\mathrm{e}}=\frac{3 y^{\mathrm{ek}}}{2}- \\
& 6\left(\frac{y^{\mathrm{ek}}\left(1+3 m / z^{2}\right) \sinh ^{2}\left(z y^{\mathrm{ek}} / 4\right)+\left[2 z^{-1} \sinh \left(z y^{\mathrm{ek}} / 2\right)-3 m y^{\mathrm{ek}}\right] \ln \cosh \left(z y^{\mathrm{ek}} / 4\right)}{\kappa a+8\left(1+3 m / z^{2}\right) \sinh ^{2}\left(z y^{\mathrm{ek}} / 4\right)-\left(24 m / z^{2}\right) \ln \cosh \left(z y^{\mathrm{ek}} / 4\right)}\right)
\end{aligned}
$$

where $m$ was defined in eq. 18 and

$$
y^{\mathrm{ek}}=\frac{e \zeta}{k T}
$$

is the dimensionless $\zeta$-potential. For aqueous solutions, $m$ is about 0.15. O'Brien [4] found that eq. 22 can be simplified by neglecting terms of order $(\kappa a)^{-1}$ as follows:

$$
\frac{3}{2} \frac{\eta e}{\varepsilon_{\mathrm{rs}} \varepsilon_{0} k T} u_{\mathrm{e}}=\frac{3}{2} y^{\mathrm{ek}}-\frac{6\left[\frac{y^{\mathrm{ek}}}{2}-\frac{\ln 2}{z}\left\{1-\exp \left(-z y^{\mathrm{ek}}\right)\right\}\right]}{2+\frac{\kappa a}{1+3 m / z^{2}} \exp \left(-\frac{z y^{\mathrm{ek}}}{2}\right)}
$$

Note that both the numerical calculations and eqs. 22 and 24 automatically account for diffuse-layer conductivity.

b.2 If a maximum in $u_{\mathrm{e}}$ (or in the apparent $\zeta$-potential deduced from the HS formula) vs. electrolyte concentration is found, the effect of stagnant-layer conduction is likely significant. For low $\zeta$-potentials, when concentration polarization is negligible, the following expression can be used [23]:

$$
u_{\mathrm{e}}=\frac{\varepsilon_{\mathrm{rs}} \varepsilon_{0}}{\eta} \zeta\left[1+\frac{D u}{1+D u}\left(\frac{k T}{e|\zeta|} \frac{2 \ln 2}{z}-1\right)\right]
$$

with $D u$ including both the stagnant- and diffuse-layer conductivities. This requires additional information about the value of $K^{\sigma \mathrm{i}}$ (see Section 3). 
c. If $\kappa a$ is low, the O'Brien and White [22] numerical calculations remain valid, but there are also several analytical approximations. For $\kappa a<1$, the Hückel-Onsager (HO) equation applies [4]:

$$
u_{\mathrm{e}}=\frac{2}{3} \frac{\varepsilon_{\mathrm{rs}} \varepsilon_{0}}{\eta} \zeta
$$

d. For the transition range between low and high $\kappa a$, Henry's formula can be applied if $\zeta$ is presumed to be low $(<50 \mathrm{mV}$; in such conditions, surface conductivity and concentration polarization are negligible). For a nonconducting sphere, Henry derived the following expression:

$$
u_{\mathrm{e}}=\frac{2}{3} \frac{\varepsilon_{\mathrm{rs}} \varepsilon_{0}}{\eta} \zeta f_{1}(\kappa a)
$$

where the function $f_{1}$ varies smoothly from 1.0 , for low values of $\kappa a$, to 1.5 as $\kappa a$ approaches infinity. Henry [24] gave two series expansions for the function $f_{1}$, one for small $\kappa a$ and one for large $\kappa a$. Ohshima [25] has provided an approximate analytical expression which duplicates the Henry expansion almost exactly. Ohshima's relation is

$$
f_{1}(\kappa a)=1+\frac{1}{2}\left[1+\left(\frac{2.5}{\kappa a[1+2 \exp (-\kappa a)]}\right)\right]^{-3}
$$

Equation 27 can be used in the calculation of the electrophoretic mobility of particles with nonzero bulk conductivity, $K_{\mathrm{p}}$. With that aim, it can be modified to read [2]

$$
\begin{aligned}
& u_{\mathrm{e}}=\frac{2}{3} \frac{\varepsilon_{\mathrm{rs}} \varepsilon_{0}}{\eta} \zeta \times F_{1}\left(\kappa a, K_{\mathrm{p}}\right) \\
& F_{1}\left(\kappa a, K_{\mathrm{p}}\right)=1+\frac{2-2 K_{\mathrm{rel}}}{1+K_{\mathrm{rel}}}\left[f_{1}(\kappa a)-1\right]
\end{aligned}
$$

with

$$
K_{\text {rel }}=\frac{K_{\mathrm{p}}}{K_{\mathrm{L}}}
$$

e. If SLC is likely for a system with low $\kappa a$, then as discussed before, $\zeta$ ceases to be the only parameter needed for a full characterization of the EDL, and additional information on $K^{\sigma}$ is required (see Section 3). Numerical calculations like those of Zukoski and Saville [9] or Mangelsdorf and White [10-12] can be used.

Figure 2a allows a comparison to be established between the predictions of the different models mentioned, for the case of spheres. For the $\kappa a$ chosen, the curvature is enough for the HS theory to be in error, the more so the higher $|\zeta|$. According to Henry's treatment, the electrophoretic mobility is lower than predicted by the simpler HS equation. Note also that Henry's theory fails for low-to-moderate $\zeta$-potentials; this is a consequence of its neglecting concentration polarization. The full O'Brien and White theory demonstrates that as $\zeta$ increases, the mobility is lower than predicted by either Henry's or HS calculations. The existence of surface conduction can account for this. In addition, for sufficiently high $\zeta$-potential, the effect of concentration polarization is a further reduction of the mobility, which goes through a maximum and eventually decreases with the increase of $\zeta$-potential.

The effect of $\kappa a$ on the $u_{\mathrm{e}}(\zeta)$ relationship is depicted in Fig. 2b. Note that the maximum is more pronounced with the larger $\kappa a$, and that the electrophoretic mobility increases (in the range of $\kappa a$ shown) with the former. Finally, Fig. 2c demonstrates the drastic change that can occur in the mobility- 

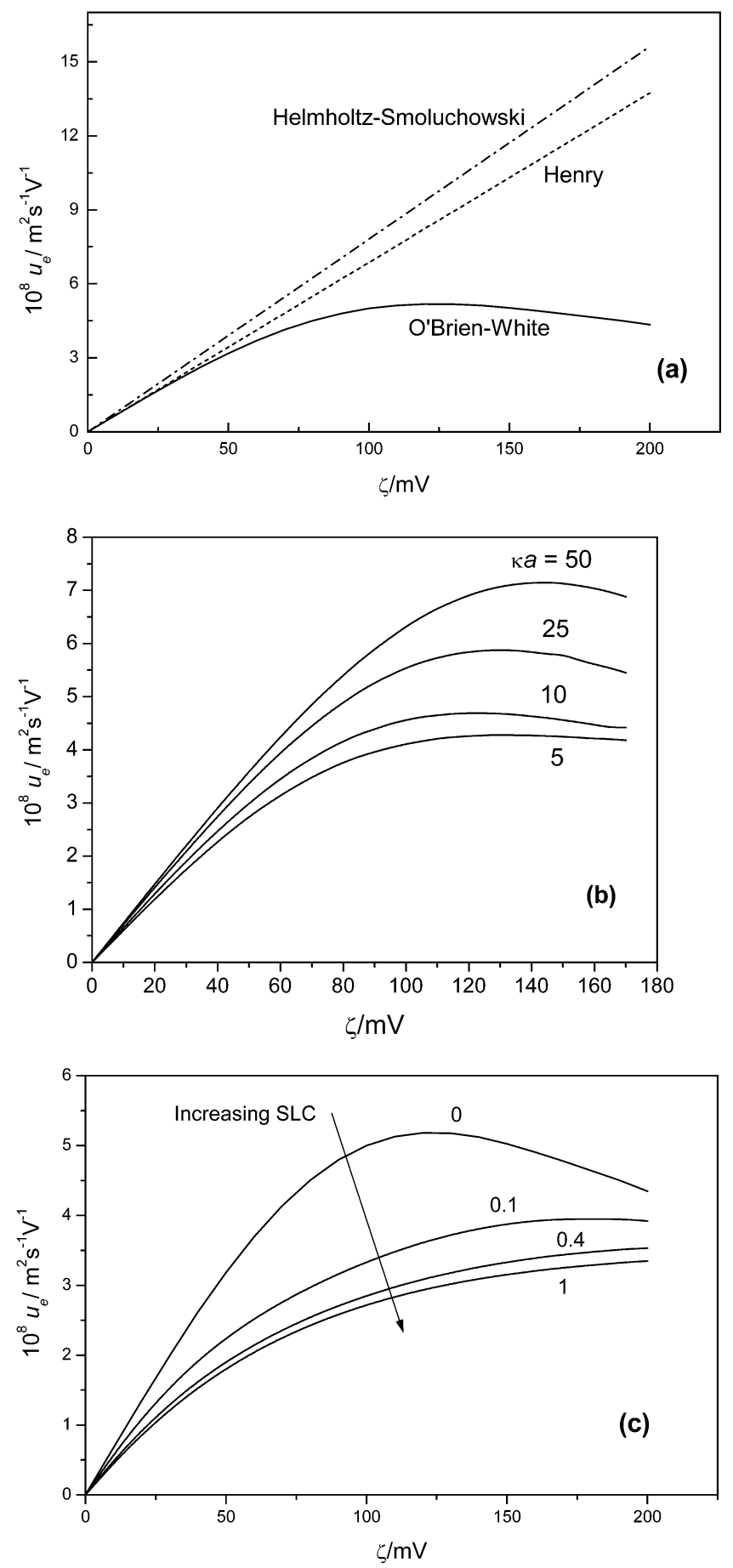

Fig. 2 (a) Electrophoretic mobility $u_{\mathrm{e}}$ plotted as a function of the $\zeta$-potential according to different theoretical treatments, all neglecting SLC: HS, O’Brien-White (full theory), Henry (no surface conductance), for $\kappa a=15$. (b) Role of $\kappa a$ on the mobility- $\zeta$ relationship (O'Brien-White theory). (c) Effect of SLC on the electrophoretic mobility- $\zeta$-potential relationship for the same suspensions as in part (a). The ratios between the diffusion coefficients of counterions in the stagnant layer and in the bulk electrolyte are indicated (the upper curve corresponds to zero SLC). 
$\zeta$-potential trends if SLC is present. This quantity always tends to decrease $u_{\mathrm{e}}$, as the total surface conductivity is increased, as compared to the case of diffuse-layer conductivity alone.

\subsubsection{Experimental techniques available: Samples}

i. $\quad$ Earlier techniques, at present seldom used in colloid science:

- $\quad$ Moving boundary [26]. In this method, a boundary is mechanically produced between the suspension and its equilibrium serum. When the electric field is applied, the migration of the solid particles provokes a displacement of the solid/liquid separation whose velocity is in fact proportional to $v_{\mathrm{e}}$. The traditional moving-boundary method contributed to a great extent to the knowledge of proteins and polyelectrolytes as well as of colloids. It inspired gel electrophoresis, presently essential in such important fields as genetic analysis.

- Mass transport electrophoresis [27]. The mass transport method is based on the fact the application of a known potential difference to the suspension causes the particles to migrate from a reservoir to a detachable collection chamber. The electrophoretic mobility is deduced from data on the amount of particles moved after a certain time, which can be determined by simply weighing the collection chamber or otherwise analyzing its contents.

ii. Microscopic (visual) microelectrophoresis

Probably the most widespread method until the 1980s, microscopic (visual) microelectrophoresis is based on the direct observation, with a suitable magnifying optics, of individual particles in their electrophoretic motion. In fact, it is not the particle that is seen, but a bright dot on a dark background, due to the Tyndall effect, that is the strong lateral light scattering of colloidal particles.

\section{Size range of samples}

The ultramicroscope is necessary for particles smaller than $0.1 \mu \mathrm{m}$. Particles about $0.5 \mu \mathrm{m}$ can be directly observed using a travelling microscope illuminated with a strong (cold) light source.

\section{Advantages and prerequisites of the technique}

- $\quad$ The particles are directly observed in their medium.

- The suspensions to be studied should be stable and dilute; if they are not, individual particles cannot be identified under the microscope. However, in dilute systems; the aggregation times are very long, even in the worst conditions, so that velocities can likely be measured.

\section{Problems involved in the technique and proposed actions to solve them}

- Its main limitations are the bias and subjectivity of the observer, who can easily select only a narrow range of velocities, which might be little representative of the true average value of the suspension. Furthermore, measurements usually take a fairly long time, and this can bring about additional problems such as Joule heating, $\mathrm{pH}$ changes, and so on. Hence, some manufacturers of commercial apparatus have modified their designs to include automatic tracking by digital image processing.

- Recall that electrophoresis is the movement of the particles with respect to the fluid, which is assumed to be at rest. However, the observed velocity is in fact relative to the instrument, and this is a source of error, as an electro-osmotic flow of liquid is also induced by the external field if the cell walls are charged, which is often the case. If the cell is open, the velocity over its section would be constant and equal to its value at the outer double-layer boundary. However, in almost all experimental set-ups, the measuring cell is closed, and the electro-osmotic counter-pressure provokes a liquid flow of Poiseuille type. The resulting velocity profile for the case of a cylindrical channel is given by [4]

$$
v_{\mathrm{L}}=v_{\mathrm{eo}}\left(2 \frac{r^{2}}{a^{2}}-1\right)
$$


where $\boldsymbol{v}_{\mathrm{eo}}$ is the electro-osmotic liquid velocity in the channel, $a$ is the capillary radius, and $r$ is the radial distance from the cylinder axis. From eq. 32, it is clear that $v_{\mathrm{L}}=0$ if $r=a / \sqrt{2}$, so that the true electrophoretic velocity will be displayed only by particles moving in a cylindrical shell placed at $0.292 a$ from the channel wall. It is easy to estimate the uncertainties associated with errors in the measuring position: if $a \sim 2 \mathrm{~mm}$ and the microscope has a focus depth of $\sim 50 \mu \mathrm{m}$, then an error of $2 \%$ in the velocity will be always present. A more accurate, although time-consuming method, consists in measuring the whole parabolic velocity profile to check for absence of systematic errors. These arguments also apply to electrophoresis cells with rectangular or square cross-sections.

Some authors (see, e.g., [28]) have suggested that a procedure to avoid this problem would be to cover the cell walls, whatever their geometry, with a layer of uncharged chemical species, for instance, polyacrylamide. However, it is possible that after some usage, the layer gets detached from the walls, and this would mask the electrophoretic velocity measured at an arbitrary depth, with an electro-osmotic contribution, the absence of which can only be ascertained by measuring $u_{\mathrm{e}}$ of standard, stable particles, which in turn remains an open problem in electrokinetics.

A more recent suggestion [29] is to perform the electrophoresis measurements in an alternating field with frequency much larger than the reciprocal of the characteristic time $\tau$ for steady electro-osmosis $(\tau \sim 1 \mathrm{~s})$, but smaller than that of steady electrophoresis $\left(\tau \sim 10^{-4} \mathrm{~s}\right)$. Under such conditions, no electro-osmotic flow can develop and hence the velocity of the particle is independent of the position in the cell.

Another way of overcoming the electro-osmosis problem is to place both electrodes providing the external field inside the cell, completely surrounded by the suspension; since no net external field acts on the charged layer close to the cell walls, the associated electroosmotic flow will not exist [30].

iii. Electrophoretic light scattering (ELS)

These are automated methods based on the analysis of the (laser) light scattered by moving particles [31-34]. They have different principles of operation [35]. The most frequently used method, known as laser Doppler velocimetry, is based on the analysis of the intensity autocorrelation function of the scattered light. The method of phase analysis light scattering (PALS) [36-38] has the adavantage of being suited for particles moving very slowly, for instance, close to their isoelectric point. The method is capable of detecting electrophoretic mobilities as low as $10^{-12} \mathrm{~m}^{2} \mathrm{~V}^{-1} \mathrm{~s}^{-1}$, that is, $10^{-4} \mu \mathrm{m} \mathrm{s}^{-1} / \mathrm{V} \mathrm{cm}^{-1}$ in practical mobility units (note that mobilities typically measurable with standard techniques must be above $\sim 10^{-9} \mathrm{~m}^{2} \mathrm{~V}^{-1} \mathrm{~s}^{-1}$ ). These techniques are rapid, and measurements can be made in a few seconds. The results obtained are very reproducible, with typical standard deviations less than $2 \%$. A small amount of sample is required for analysis, often a few millilitres of a suitable dispersion. However, dilution of the sample may be required, and therefore the sample preparation technique becomes very important.

\section{Samples that can be studied}

a. Sample composition

Measurements can be made of any colloidal dispersion where the continuous phase is a transparent liquid and the dispersed phase has a refractive index which differs from that of the continuous phase.

b. Size range of samples

The lower size limit is dependent upon the sample concentration, the refractive index difference between disperse and continuous phase, and the quality of the optics and performance of the instrument. Particle sizes down to $5 \mathrm{~nm}$ can be measured under optimum conditions. 
The upper size limit is dependent upon the rate of sedimentation of particles (which is related to particle size and density). ELS methods are inherently directional in their measurement plane. Hence, for a horizontal field, samples can be measured while they are sedimenting. Measurement is possible so long as there are particles present in the detection volume. Typically, measurements are possible for particles with diameters below $30 \mu \mathrm{m}$.

c. Sample conductivity

The conductivity of samples that can be measured ranges from that of particles dispersed in deionized water up to media containing greater than physiological saline. In high salt concentration, the Joule heating of the sample will affect the particle mobility, and thermostating of the cell is not at all easy. Reduction of the applied voltage decreases this effect, but will also reduce the resolution obtainable from the measurement.

The presence of some ions in the medium is recommended (e.g., $10^{-4} \mathrm{~mol} / \mathrm{L} \mathrm{NaCl}$ ) as this will stabilize the field in the cell and will improve the repeatability of measurements. Furthermore, some salt is always needed anyway because otherwise the double layer becomes ill-defined.

d. Sample viscosity

There is no particular limit as to the viscosity range of samples that can be measured. But it must be emphasized that increasing the viscosity of the medium will reduce the mobility of the particles and may require longer observation times, with the subsequent increased risk of Joule heating.

e. Permittivity

Measurements in a large variety of solvents are possible, depending on the instrument configuration.

f. Fluorescence

Sample fluorescence results in a reduction in the signal-to-noise ratio of the measurement. In severe cases, this may completely inhibit measurements.

\section{Sample preparation}

Many samples will be too concentrated for direct measurement and will require dilution. How this dilution is carried out is critical. The aim of sample preparation is to preserve the existing state of the particle surface during the process of dilution. One way to ensure this is by filtering or gently centrifuging some clear liquid from the original sample and using this to dilute the original concentrated sample. In this way, the equilibrium between surface and liquid is perfectly maintained. If extraction of a supernatant is not possible, then just letting a sample naturally sediment and using the fine particles left in the supernatant is a good alternative method. The possibility also exists of dialyzing the concentrate against a solution of the desired ionic composition. Another method is to imitate the original medium as closely as possible. This should be done with regard to $\mathrm{pH}$, concentration of each ionic species in the medium, and concentration of any other additive that might be present.

However, attention must be paid to the possible modification of the surface compositon upon dilution, particularly when polymers or polyelectrolytes are in solution [39]. Also, if the particles are positively charged, care must be taken to avoid long storage in glass containers, as dissolution of glass can lead to adsorption of negatively charged species on the particles. For emulsion systems, dilution is always problematic, because changing the phase volume ratio may alter the surface properties due to differential solubility effects.

\section{Ranges of electrolyte and particle concentration that can be investigated}

Microelectrophoresis is a technique where samples must be dilute enough for particles not to interfere with each other. For any system under investigation, it is recommended that an experiment should be done to check the effect of concentration on the mobility. The concentration range which can be studied will depend upon the suitability of the sample (e.g., size, refractive index) 
and the optics of the instrument. By way of example, a 200-nm polystyrene latex standard (particle refractive index 1.59, particle absorbance 0.001 ) dispersed in water (refractive index 1.33) can be measured at a solids concentration ranging from $2 \times 10^{-3}$ to $1 \times 10^{-6} \mathrm{~g} / \mathrm{cm}^{3}$.

Standard samples for checking correct instrument operation

Microelectrophoresis ELS instruments are constructed from basic physical principles and as such need not be calibrated. The correctness of their operation can only be verified by measuring a sample of which the $\zeta$-potential is known. A pioneering study in this direction was performed in 1970 by a group of Japanese surface and colloid chemists, forming a committee under the Division of Surface Chemistry in the Japan Oil Chemists Society [6,39]. This group measured and compared $\zeta$-potentials of samples of titanium dioxide, silver iodide, silica, microcapsules, and some polymer latexes. The study involved different devices in nine laboratories, and concluded that the negatively charged PSSNa (polystyrene-sodium $p$-vinylbenzenesulfonate copolymer) particles prepared as described in [40] could be a very useful standard, providing reliable and reproducible mobility data. Currently, there is no negative $\zeta$-potential standard available from the U.S. National Institute of Standards and Technology (NIST).

A positively charged sample available from NIST is Standard Reference Material (SRM) 1980. It contains a $500 \mathrm{mg} / \mathrm{L}$ goethite $(\alpha-\mathrm{FeOOH})$ suspension saturated with $100 \mu \mathrm{mol} / \mathrm{g}$ phosphate in a $5 \times 10^{-2} \mathrm{~mol} / \mathrm{L}$ sodium perchlorate electrolyte solution at a $\mathrm{pH}$ of 2.5 . When prepared according to the procedure supplied by NIST, the certified value and uncertainty for the positive electrophoretic mobility of SRM1980 is $2.53 \pm 0.12 \mu \mathrm{m} \mathrm{s}^{-1} / \mathrm{V} \mathrm{cm}^{-1}$. This will give a $\zeta$-potential of $+32.0 \pm 1.5 \mathrm{mV}$ if the HS equation (eq. 7) is used.

\subsection{Streaming current and streaming potential}

\subsubsection{Operational definitions; recommended symbols and terminology; conversion of the measured quantities into $\zeta$-potential}

The phenomena of streaming current and streaming potential occur in capillaries and plugs and are caused by the charge displacement in the EDL as a result of an applied pressure inducing the liquid phase to move tangentially to the solid. The streaming current can be detected directly by measuring the electric current between two positions, one upstream and the other downstream. This can be carried out via nonpolarizable electrodes, connected to an electrometer of sufficiently low internal resistance.

Streaming current

The first quantity of interest is the streaming current per pressure drop, $I_{\mathrm{str}} / \Delta p$ (SI units: $\mathrm{A} \mathrm{Pa}^{-1}$ ), where $I_{\text {str }}$ is the measured current, and $\Delta p$ the pressure drop. The relation between $I_{\text {str }} / \Delta p$ and $\zeta$-potential has been found for a number of cases:

a. If $\kappa a \gg 1$ ( $a$ is the capillary radius), the HS formula can be used

$$
\frac{I_{\mathrm{str}}}{\Delta p}=-\frac{\varepsilon_{\mathrm{rs}} \varepsilon_{0} \zeta}{\eta} \frac{A_{\mathrm{c}}}{L}
$$

where $A_{\mathrm{c}}$ is the capillary cross-section, and $L$ its length. If instead of a single capillary, the experimental system is a porous plug or a membrane, eq. 33 remains approximately valid, provided that $\kappa a \gg 1$ everywhere in the pore walls. In the case of porous plugs, attention has to be paid to the fact that a plug is not a system of straight parallel capillaries, but a random distribution of particles with a resulting porosity and tortuosity, for which an equivalent capillary length and crosssection is just a simplified model. In addition, the use of eq. 33 requires that the conduction current in the system is determined solely by the bulk conductivity of the supporting solution. It often happens that surface conductivity is important, and, besides that, the ions in the plug behave with a lower mobility than in solution. 
$\mathrm{A}_{c} / L$ can be estimated experimentally as follows [40,41]. Measure the resistance, $R_{\infty}$, of the plug or capillary wetted by a highly concentrated (above $10^{-2} \mathrm{~mol} / \mathrm{L}$, say) electrolyte solution, with conductivity $K_{\mathrm{L}}^{\infty}$. Since for such a high ionic strength the double-layer contribution to the overall conductivity is negligible, we may write

$$
\frac{A_{\mathrm{c}}}{L}=\frac{1}{K_{\mathrm{L}}^{\infty} R_{\infty}}
$$

In addition, theoretical or semi-empirical models exist that relate the apparent values of $A_{\mathrm{c}}$ and $L$ (external dimensions of the plug) to the volume fraction, $\phi$, of solids in the plug. For instance, according to [42]

$$
\frac{A_{\mathrm{c}}}{L}=\frac{A_{\mathrm{c}}^{\text {ap }}}{L^{\text {ap }}} \exp (B \phi)
$$

where $B$ is an empirical constant that can be experimentally determined by measuring the electro-osmotic volume flow for different plug porosities. In eq. $35, L^{\text {ap }}$ and $A_{\mathrm{c}}$ ap are the apparent (externally measured) length and cross-sectional area of the plug, respectively. An alternative expression was proposed in [43]:

$$
\frac{A_{\mathrm{c}}}{L}=\frac{A_{\mathrm{c}}^{\mathrm{ap}}}{L^{\mathrm{ap}}} \phi_{\mathrm{L}}^{-5 / 2}
$$

where $\phi_{\mathrm{L}}$ is the volume fraction of liquid in the plug (or void volume fraction). Other estimates of $A_{c} / L$ can be found in [44-46].

For the case of a close packing of spheres, theoretical treatments are available involving the calculation of streaming current using cell models. No simple expressions can be given in this case; see [3,47-52] for details.

b. If $\kappa a$ is intermediate ( $\kappa a \approx 1-10$, say), the HS equation is not valid. For low $\zeta$, curvature effects can be corrected by means of the Burgen and Nakache theory [4,49]:

$$
\frac{I_{\mathrm{str}}}{\Delta p}=\frac{\varepsilon_{\mathrm{rs}} \varepsilon_{0} \zeta}{\eta} \frac{A_{\mathrm{c}}}{L}[1-G(\kappa a)]
$$

where

$$
G(\kappa a)=\frac{\tanh (\kappa a)}{\kappa a}
$$

for slit-shaped capillaries ( $2 a$ corresponds in this case to the separation of the parallel solid walls). In the case of cylindrical capillaries of radius $a$, the calculattion was first carried out by Rice and Whitehead [50]. They found that the function $G(\kappa a)$ in eq. 37 reads

$$
G(\kappa a)=\frac{2 I_{1}(\kappa a)}{\kappa a I_{0}(\kappa a)}
$$

where $I_{0}$ and $I_{1}$ are the zeroth- and first-order modified Bessel functions of the first kind, respectively. Fig. 3 illustrates the importance of this curvature correction. 


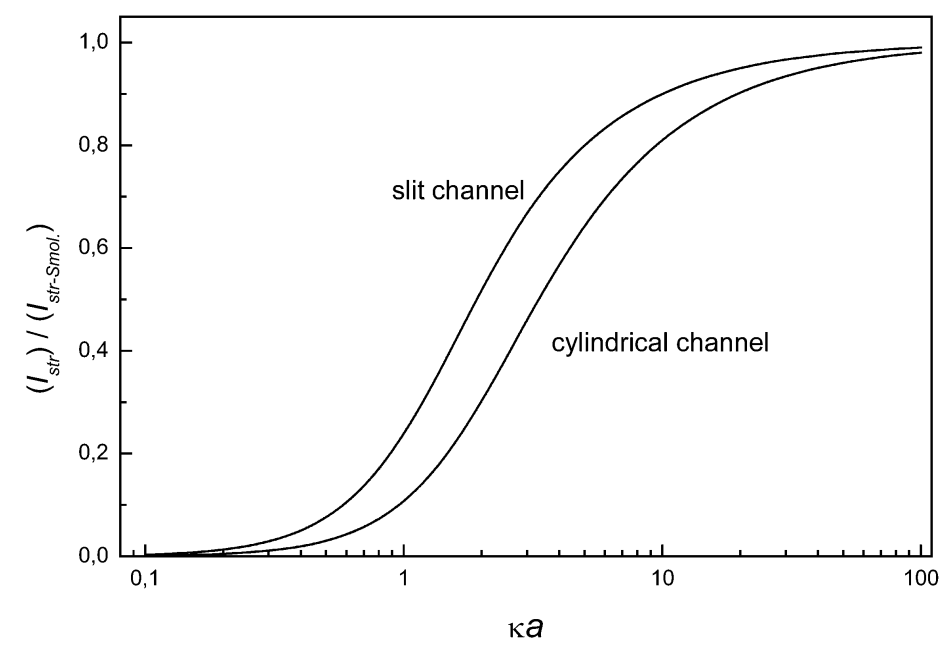

Fig. 3 Streaming current divided by the applied pressure difference, eqs. 37 and 39, relative to the Smoluchowski formula, eq. 33 , plotted as a function of the product $\kappa a$ ( $a$ : capillary radius, or slit half-width) for slit- and cylindrical-shaped capillaries.

c. If the $\zeta$-potential is not low and $\kappa a$ is small, no simple expression for $I_{\text {str }}$ can be given, and only numerical procedures are available [52].

Streaming potential

The streaming potential difference (briefly, streaming potential) $U_{\text {str }}$ can be measured between two electrodes, upstream and downstream in the liquid flow, connected via a high-input impedance voltmeter. The quantity of interest is, in this case, the ratio between the streaming potential and the pressure drop, $U_{\mathrm{str}} \mathrm{J} \Delta p\left(\mathrm{~V} \mathrm{~Pa}^{-1}\right)$. The conversion into $\zeta$-potentials can be realized in a number of cases.

a. If $\kappa a \gg>1$ and surface conduction can be neglected, the HS formula can be used:

$$
\frac{U_{\mathrm{str}}}{\Delta p}=\frac{\varepsilon_{\mathrm{rs}} \varepsilon_{0} \zeta}{\eta} \frac{1}{K_{\mathrm{L}}}
$$

b. The most frequent case (except for high ionic strengths, or high $K_{\mathrm{L}}$ ) is that surface conductance, $K^{\sigma}$, is significant. Then the following equation should be used:

$$
\frac{U_{\mathrm{str}}}{\Delta p}=\frac{\varepsilon_{\mathrm{rs}} \varepsilon_{0} \zeta}{\eta} \frac{1}{K_{\mathrm{L}}(1+2 D u)}
$$

where $D u$ is given by eqs. 12 and 20 .

An empirical way of taking into account the existence of surface conductivity is to measure the resistance $R_{\infty}$ of the plug or capillary in a highly concentrated electrolyte solution of conductivity $K_{\mathrm{L}}^{\infty}$. As for such a solution, $D u$ is negligible, one can write

$$
K_{\mathrm{L}}^{\infty} R_{\infty}=\left(K_{\mathrm{L}}+\frac{2 K^{\sigma}}{a}\right) R_{\mathrm{s}}
$$

where $R_{\mathrm{S}}$ is the resistance of the plug in the solution under study, of conductivity $K_{\mathrm{L}}$. Now, eq. 41 can be approximated by 


$$
\frac{U_{\mathrm{str}}}{\Delta p}=\frac{\varepsilon_{\mathrm{rs}} \varepsilon_{0} \zeta}{\eta} \frac{R_{\mathrm{s}}}{K_{\mathrm{L}}^{\infty} R_{\infty}}
$$

c. If $\kappa a$ is intermediate $(\kappa a \sim 1 \ldots 10)$ and the $\zeta$-potential is low, Rice and Whitehead's corrections are needed [50]. For a cylindrical capillary, the result is

$$
\frac{U_{\mathrm{str}}}{\Delta p}=\frac{\varepsilon_{\mathrm{rs}} \varepsilon_{0} \zeta}{\eta} \frac{R_{\mathrm{s}}}{K_{\mathrm{L}}^{\infty} R_{\infty}} \frac{1-\frac{2 I_{1}(\kappa a)}{\kappa a I_{0}(\kappa a)}}{1-\beta\left[1-\frac{2 I_{1}(\kappa a)}{\kappa a I_{0}(\kappa a)}-\frac{I_{1}^{2}(\kappa a)}{I_{0}^{2}(\kappa a)}\right]}
$$

where

$$
\beta=\frac{\left(\varepsilon_{\mathrm{rs}} \varepsilon_{0} \kappa \zeta\right)^{2}}{\eta} \frac{R_{\mathrm{s}}}{K_{\mathrm{L}}^{\infty} R_{\infty}}
$$

Figure 4 illustrates some results that can be obtained by using eq. 44 .

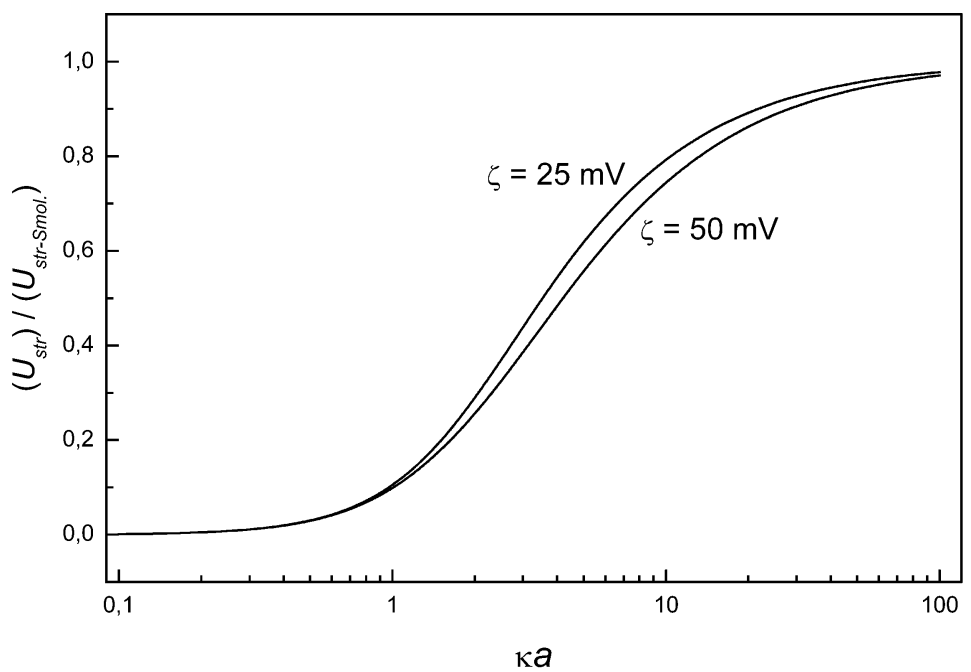

Fig. 4 Streaming potential divided by the applied pressure difference, eq. 44, relative to its Smoluchowski value, eq. 40 , as a function of the product $\kappa a$ ( $a$ : capillary radius), for the $\zeta$-potentials indicated. Surface conductance is neglected.

d. As in the case of streaming current, for high $\zeta$-potentials, only numerical methods are available (see, e.g., [53] for details).

In practice, instead of potential or current measurements for just one driving pressure, the streaming potential and streaming current are mostly measured at various pressure differences applied in both directions across the capillary system, and the slopes of the functions $U_{\text {str }}=U_{\text {str }}(\Delta p)$ and $I_{\text {str }}=I_{\text {str }}(\Delta p)$ are used to calculate the $\zeta$-potential. This makes it possible to detect electrode asymmetry effects and correct for them. It is also advisable to verify that the $\Delta p$ dependencies are linear and pass through the origin. 


\subsubsection{Samples that can be studied}

Streaming potential/current measurements can be applied to study macroscopic interfaces of materials of different shape. Single capillaries made of flat sample surfaces (rectangular capillaries) and cylindrical capillaries can be used to produce micro-channels for streaming potential/current measurements. Further, parallel capillaries and irregular capillary systems such as fiber bundles, membranes, and particle plugs can also be studied. Recall, however, the precautions already mentioned in connection with the interpretation of results in the case of plugs of particles. Other effects, including temperature gradients, Donnan potentials, or membrane potential can contribute to the observed streaming potential or electro-osmotic flow. An additional condition is the constancy of the capillary geometry during the course of the experiment. Reversibility of the signal upon variations in the sign and magnitude of $\Delta p$ is a criterion for such constancy.

Most of the materials studied so far by streaming potential/current measurements, including synthetic polymers and inorganic non-metals, are insulating. Either bulk materials or thin films on top of carriers can be characterized. In addition, in some cases, semiconductors [54] and even bulk metals [55] have been studied, proving the general feasibility of the experiment.

Note that streaming potential/current measurements on samples of different geometries (flat plates, particle plugs, fiber bundles, cylindrical capillaries,...) each require their own set-up.

\subsubsection{Sample preparation}

The samples to be studied by streaming potential/current measurements have to be mechanically and chemically stable in the aqueous solutions used for the experiment. First, the geometry of the plug must be consolidated in the measuring cell. This can be checked by rinsing with the equilibrium liquid through repeatedly applying $\Delta p$ in both directions until finding a constant signal. Another issue to consider is the necessity that the solid has reached chemical equilibrium with the permeating liquid; this may require making the plug from a suspension of the correct composition, followed by rinsing. Checking that the experimental signal does not change during the course of measurement may be a good practice. The presence or formation of air bubbles in the capillary system has to be avoided.

Standard samples

No standard samples have been developed specifically so far for streaming potential/current measurements, although several materials have been frequently analyzed and may, therefore, serve as potential reference samples [56,57].

Range of electrolyte concentrations

From the operational standpoint, there is no lower limit to the ionic strength of the systems to be investigated by these methods, although in the case of narrow channels, very low ionic strengths require effective electrical insulation of the set-up in order to prevent short-circuiting. However, such low ionic strength values can only be attained if the solid sample is extremely pure and insoluble. The upper value of electrolyte concentration depends on the sensitivity of the electrometer and on the applied pressure difference; usually, solutions above $10^{-1} \mathrm{~mol} / \mathrm{L}$ of 1-1 charge-type electrolyte are difficult to measure by the present techniques.

\subsection{Electro-osmosis}

\subsubsection{Operational definitions; recommended symbols and terminology; conversion of the measured quantities into $\zeta$-potential}

In electro-osmosis, a flow of liquid is produced when an electric field $\boldsymbol{E}$ is applied to a charged capillary or porous plug immersed in an electrolyte solution. If $\kappa a>>1$ everywhere at the solid/liquid interface, far from that interface the liquid will attain a constant (i.e., independent of the position in the channel) velocity (the electro-osmotic velocity) $v_{\text {eo }}$, given by eq. 4 . If such a velocity cannot be measured, the convenient physical quantity becomes the electro-osmotic flow rate, $Q_{\mathrm{eo}}\left(\mathrm{m}^{3} \mathrm{~s}^{-1}\right)$, given by 


$$
Q_{\mathrm{eo}}=\iint_{A_{\mathrm{c}}} v_{\mathrm{eo}} \cdot \mathrm{d} \boldsymbol{S}
$$

where $\mathrm{d} \boldsymbol{S}$ is the elementary surface vector at the location in the channel where the fluid velocity is $\boldsymbol{v}_{\mathrm{eo}}$. The counterparts of $Q_{\mathrm{eo}}$ are $Q_{\mathrm{eo}, E}$ (flow rate divided by electric field) and $Q_{\mathrm{eo}, I}$ (flow rate divided by current). These are the quantities that can be related to the $\zeta$-potential. As before, several cases can be distinguished:

a. If $\kappa a \gg>1$ and there is no surface conduction:

$$
\begin{gathered}
Q_{\mathrm{eo}, E} \equiv \frac{Q_{\mathrm{eo}}}{E}=-\frac{\varepsilon_{\mathrm{rs}} \varepsilon_{0} \zeta}{\eta} A_{\mathrm{c}} \\
Q_{\mathrm{e}, I} \equiv \frac{Q_{\mathrm{eo}}}{I}=-\frac{\varepsilon_{\mathrm{rs}} \varepsilon_{0} \zeta}{\eta} \frac{1}{K_{\mathrm{L}}}
\end{gathered}
$$

b. With surface conduction, the expression for $Q_{\mathrm{eo}, E}$ is as in eq. 47 , and that for $Q_{\mathrm{eo}, I}$ is

$$
Q_{\mathrm{eo}, I}=-\frac{\varepsilon_{\mathrm{rs}} \varepsilon_{0} \zeta}{\eta} \frac{1}{K_{\mathrm{L}}(1+2 D u)}
$$

In eq. 48 , the empirical approach for the estimation of $D u$ can be followed:

$$
Q_{\mathrm{e}, I}=-\frac{\varepsilon_{\mathrm{rs}} \varepsilon_{0} \zeta}{\eta} \frac{R_{\mathrm{s}}}{K_{\mathrm{L}}^{\infty} R_{\infty}}
$$

c. Low $\zeta$-potential, finite surface conduction, and arbitrary capillary radius [46]:

$$
\begin{aligned}
& Q_{\mathrm{eo}, E}=-\frac{\varepsilon_{\mathrm{rs}} \varepsilon_{0} \zeta}{\eta} A_{\mathrm{c}}[1-G(\kappa a)] \\
& Q_{\mathrm{e}, I}=-\frac{\varepsilon_{\mathrm{rs}} \varepsilon_{0} \zeta}{\eta} \frac{[1-G(\kappa a)]}{K_{\mathrm{L}}(1+2 D u)} \cong-\frac{\varepsilon_{\mathrm{rs}} \varepsilon_{0} \zeta}{\eta} \frac{R_{\mathrm{s}}[1-G(\kappa a)]}{K_{\mathrm{L}}^{\infty} R_{\infty}}
\end{aligned}
$$

where the function $G(\kappa a)$ is given by eq. 38 .

d. When $\zeta$ is high and the condition $\kappa a \gg>1$ is not fulfilled, no simple expression can be given for $Q_{\text {eo. }}$.

As in the case of streaming potential and current, the procedures described can be also applied to either plugs or membranes. If the electric field $\boldsymbol{E}$ is the independent variable (see eq. 47), then $A_{\mathrm{c}}$ must be estimated. In that situation, the recommendations suggested in Section 3.2.1 can be used, since eq. 47 can be written as

$$
\frac{Q_{\mathrm{eo}, E}}{\Delta V_{\mathrm{ext}}}=-\frac{\varepsilon_{\mathrm{rs}} \varepsilon_{0} \zeta}{\eta} \frac{A_{\mathrm{c}}}{L}
$$

where $\Delta V_{\text {ext }}$ is the applied potential difference.

\subsubsection{Samples that can be studied}

The same samples as with streaming current/potential, see Section 4.2.2.

\subsubsection{Sample preparation and standard samples}

See Section 4.2.3, referring to streaming potential/current determination. 


\subsection{Experimental determination of surface conductivity}

Surface conductivities are excess quantities and cannot be directly measured. There are, in principle, three methods to estimate them.

i. In the case of plugs, measure the plug conductivity $K_{\text {plug }}$ as a function of $K_{\mathrm{L}}$. The latter can be changed by adjusting the electrolyte concentration. The plot of $K_{\text {plug }}$ vs. $K_{\mathrm{L}}$ has a large linear range which can be extrapolated to $K_{\mathrm{L}}=0$ where the intercept represents $K^{\sigma}$. This method requires a plug and seems relatively straightforward.

ii. For capillaries, deduce $K^{\sigma}$ from the radius dependence of the streaming potential, using eq. 41 and the definition of $D u$ (eq. 12). This method is rather direct, but requires a range of capillaries with different radii, but identical surface properties [58,59].

iii. Utilize the observation that, when surface conductivity is not properly accounted for, different electrokinetic techniques may give different values for the $\zeta$-potential of the same material under the same solution conditions. Correct the theories by inclusion of the appropriate surface conductivity, and find in this way the value of $K^{\sigma}$ that harmonizes the $\zeta$-potential. This method requires insight into the theoretical backgrounds [60,61], and it works best if the two electrokinetic techniques have a rather different sensitivity for surface conduction (such as electrophoresis and LFDD).

In many cases, it is found that the surface conductivity obtained in one of these ways exceeds $K^{\sigma d}$, sometimes by orders of magnitude. This means that $K^{\sigma \mathrm{i}}$ is substantial. The procedure for obtaining $K^{\sigma \mathrm{i}}$ consists of subtracting $K^{\sigma \mathrm{d}}$ from $K^{\sigma}$. For $K^{\sigma \mathrm{d}}$, Bikerman's equation (eq. 17) can be used. The method is not direct because this evaluation requires the $\zeta$-potential, which is one of the unknowns; hence, iteration is required.

\subsection{Dielectric dispersion}

\subsubsection{Operational definitions; recommended symbols and terminology; conversion of the measured quantities into $\zeta$-potential}

The phenomenon of dielectric dispersion in colloidal suspensions involves the study of the dependence on the frequency of the applied electric field of the electric permittivity and/or the electric conductivity of disperse systems. When dealing with such heterogeneous systems as colloidal dispersions, these quantities are defined as the electric permittivity and conductivity of a sample of homogeneous material, that when placed between the electrodes of the measuring cell, would have the same resistance and capacitance as the suspension. The dielectric investigation of dispersed systems involves determinations of their complex permittivity, $\varepsilon^{*}(\omega)\left(\mathrm{F} \mathrm{m}^{-1}\right)$ and complex conductivity $K^{*}(\omega)\left(\mathrm{S} \mathrm{m}^{-1}\right)$ as a function of the frequency $\omega\left(\mathrm{rad} \mathrm{s}^{-1}\right)$ of the applied ac field. These quantities are related to the volume, surface, and geometrical characteristics of the dispersed particles, the nature of the dispersion medium, and also to the concentration of particles, expressed either in terms of volume fraction, $\phi$ (dimensionless) or number concentration $N\left(\mathrm{~m}^{-3}\right)$.

It is common to use the relative permittivity $\varepsilon_{\mathrm{r}}^{*}(\omega)$ (dimensionless), instead of the permittivity

$$
\varepsilon^{*}(\omega)=\varepsilon_{\mathrm{r}}^{*}(\omega) \varepsilon_{0}
$$

$\varepsilon_{0}$ being the permittivity of vacuum. $K^{*}(\omega)$ and $\varepsilon^{*}(\omega)$ are not independent quantities:

$$
K^{*}(\omega)=K_{\mathrm{DC}}-i \omega \varepsilon^{*}(\omega)=K_{\mathrm{DC}}-i \omega \varepsilon_{0} \varepsilon_{\mathrm{r}}^{*}(\omega)
$$


or, equivalently,

$$
\begin{aligned}
& \operatorname{Re}[K(\omega)]=K_{\mathrm{DC}}+\omega \varepsilon_{0} \operatorname{Im}\left[\varepsilon_{\mathrm{r}}^{*}(\omega)\right] \\
& \operatorname{Im}\left[K^{*}(\omega)\right]=-\omega \varepsilon_{0} \operatorname{Re}\left[\varepsilon_{\mathrm{r}}^{*}(\omega)\right]
\end{aligned}
$$

where $K_{\mathrm{DC}}$ is the direct-current (zero frequency) conductivity of the system.

The complex conductivity $K^{*}$ of the suspension can be expressed as

$$
K^{*}(\omega)=K_{\mathrm{L}}+\delta K^{*}(\omega)
$$

where $\delta K^{*}(\omega)$ is usually called conductivity increment of the suspension. Similarly, the complex dielectric constant of the suspension can be written in terms of a relative permittivity increment or, briefly, dielectric increment $\delta \varepsilon_{\mathrm{r}}^{*}(\omega)$ :

$$
\varepsilon_{\mathrm{r}}^{*}(\omega)=\varepsilon_{\mathrm{rs}}+\delta \varepsilon_{\mathrm{r}}^{*}(\omega)
$$

As in homogeneous materials, the electric permittivity is the macroscopic manifestation of the electrical polarizability of the suspension components. Mostly, more than one relaxation frequency is observed, each associated with one of the various mechanisms contributing to the system's polarization. Hence, the investigation of the frequency dependence of the electric permittivity or conductivity allows us to obtain information about the characteristics of the disperse system that are responsible for the polarization of the particles.

The frequency range over which the dielectric dispersion of suspensions in electrolyte solutions is usually measured extends between $0.1 \mathrm{kHz}$ and several hundred MHz. In order to define in this frame the low-frequency and high-frequency ranges, it is convenient to introduce an important concept dealing with a point in the frequency scale. This frequency corresponds to the reciprocal of the Maxwell-Wagner-O'Konski relaxation time $\tau_{\mathrm{MWO}}$

$$
\omega_{\mathrm{MWO}} \equiv \frac{1}{\tau_{\mathrm{MWO}}} \equiv \frac{(1-\phi) K_{\mathrm{p}}+(2+\phi) K_{\mathrm{L}}}{\varepsilon_{0}\left[(1-\phi) \varepsilon_{\mathrm{rp}}+(2+\phi) \varepsilon_{\mathrm{rs}}\right] \varepsilon_{\mathrm{rs}}}
$$

and it is called the Maxwell-Wagner-O'Konski relaxation frequency. In eq. 57, $\varepsilon_{\text {rp }}$ is the relative permittivity of the dispersed particles. For low volume fractions and low permittivity of the particles $\left(\varepsilon_{\mathrm{rp}}<<\varepsilon_{\mathrm{rs}}\right)$, this expression reduces to

$$
\tau_{\mathrm{MWO}} \approx \frac{\varepsilon_{\mathrm{rs}} \varepsilon_{0}}{K_{\mathrm{L}}} \approx \frac{1}{2 D \kappa^{2}}
$$

where $D$ is the mean diffusion coefficient of ions in solution. The last term in eq. 58 suggests that $\tau_{\text {MWO }}$ roughly corresponds in this case to the time needed for ions to diffuse a distance of the order of one Debye length. In fact, in such conditions $\tau_{\mathrm{MWO}}$ equals $\tau_{\mathrm{el}}$, the so-called relaxation time of the electrolyte solution. It is a measure of the time required for charges in the electrolyte solution to recover their equilibrium distribution after ceasing an external perturbation. Its role in the time domain is similar to the role of $\kappa^{-1}$ in assessing the double-layer thickness.

The low-frequency range can be defined by the inequality

$$
\omega<\omega_{\mathrm{MWO}}
$$

For these frequencies, the characteristic value of the conduction current density in the electrolyte solution significantly exceeds the displacement current density, and the spatial distribution of the local elec- 
tric fields in the disperse system is mainly determined by the distribution of ionic currents. The frequency dependence shown by the permittivity of colloidal systems in this frequency range is known as low-frequency dielectric dispersion or LFDD.

In the high-frequency range, determined by the inequality

$\omega>\omega_{\mathrm{MWO}}$

the characteristic value of the displacement current density exceeds that of conduction currents, and the space distribution of the local electric fields is determined by polarization of the molecular dipoles, rather than by the distribution of ions.

\subsubsection{Dielectric dispersion and ל-potential: Models}

a. Middle-frequency range: Maxwell-Wagner-O'Konski relaxation

There are various mechanisms for the polarization of a heterogeneous material, each of which is always associated with some property that differs between the solid, the liquid, and their interface. The most widely known mechanism of dielectric dispersion, the Maxwell-Wagner dispersion, occurs when the two contacting phases have different conductivities and electric permittivities. If the ratio $\varepsilon_{\mathrm{rp}} / K_{\mathrm{p}}$ is different from that of the dispersion medium, i.e., if

$$
\frac{\varepsilon_{\mathrm{rp}}}{K_{\mathrm{p}}} \neq \frac{\varepsilon_{\mathrm{rs}}}{K_{\mathrm{L}}}
$$

the conditions of continuity of the normal components of the current density and the electrostatic induction on both sides of the surface are inconsistent with each other. This results in the formation of free ionic charges near the surface. The finite time needed for the formation of such a free charge is in fact responsible for the Maxwell-Wagner dielectric dispersion.

In the Maxwell-Wagner model, no specific properties are assumed for the surface, which is simply considered as a geometrical boundary between homogeneous phases. The Maxwell-Wagner model was generalized by O'Konski [61], who first took the surface conductivity $K^{\sigma}$ explicitly into account. In his treatment, the conductivity of the particle is modified to include the contributions of both the solid material and the excess surface conductivity. This effective conductivity will be called $K_{\text {pef }}$ :

$$
K_{\text {pef }}=K_{\mathrm{p}}+\frac{2 K^{\sigma}}{a}
$$

Both the conductivity and the dielectric constant can be considered as parts of a complex electric permittivity of any of the system's components. Thus, for the dispersion medium

$$
\varepsilon_{\mathrm{rs}}^{*}=\varepsilon_{\mathrm{rs}}-i \frac{K_{\mathrm{L}}}{\omega \varepsilon_{0}}
$$

and for the particle

$$
\varepsilon_{\mathrm{rp}}^{*}=\varepsilon_{\mathrm{rp}}-i \frac{K_{\mathrm{pef}}}{\omega \varepsilon_{0}}
$$

In terms of these quantities, the Maxwell-Wagner-O' Konski theory gives the following expression for the complex dielectric constant of the suspension:

$$
\varepsilon_{\mathrm{r}}^{*}=\varepsilon_{\mathrm{rs}}^{*} \frac{\varepsilon_{\mathrm{rp}}^{*}+2 \varepsilon_{\mathrm{rs}}^{*}+2 \phi\left(\varepsilon_{\mathrm{rp}}^{*}-\varepsilon_{\mathrm{rs}}^{*}\right)}{\varepsilon_{\mathrm{rp}}^{*}+2 \varepsilon_{\mathrm{rs}}^{*}-\phi\left(\varepsilon_{\mathrm{rp}}^{*}-\varepsilon_{\mathrm{rs}}^{*}\right)}
$$


b. Low-frequency range: dilute suspensions of nonconducting spherical particles with $\kappa a \gg>1$, and negligible $K^{\sigma \mathrm{i}}$

At moderate or high $\zeta$-potentials, mobile counterions are more abundant than coions in the EDL. Therefore, the contribution of the counterions and the coions to surface currents in the EDL differs from their contribution to currents in the bulk solution. Such difference gives rise to the existence of a field-induced perturbation of the electrolyte concentration, $\delta c(\boldsymbol{r})$, in the vicinity of the polarized particle. The ionic diffusion caused by $\delta c(\boldsymbol{r})$ provokes a low-frequency dependence of the particle's dipole coefficient, $C_{0}^{*}$ (see below). This is the origin of the LFDD ( $\alpha$-dispersion) displayed by colloidal suspensions. Recall that the dipole coefficient relates the dipole moment $\boldsymbol{d}^{*}$ to the applied field $\boldsymbol{E}$. For the case of a spherical particle of radius $a$, the dipole coefficient is defined through the relation

$$
\boldsymbol{d}^{*}=4 \pi \varepsilon_{\mathrm{rs}} \varepsilon_{0} a^{3} C_{0}^{*} \boldsymbol{E}
$$

The calculation of this quantity proves to be essential for evaluation of the dielectric dispersion of the suspension [63-65]. A model for the calculation of the low-frequency conductivity increment $\delta K^{*}(\omega)$ and relative permittivity increment $\delta \varepsilon_{\mathrm{r}}^{*}(\omega)$ from the dipole coefficient $C_{0}^{*}$ when $\kappa a \gg>1$ is described in Appendix I. There it is shown that, in the absence of SLC, the only parameter of the solid/liquid interface that is needed to account for LFDD is the $\zeta$-potential.

The overall behavior is illustrated in Fig. 5 for a dilute dispersion of spherical nonconducting particles $\left(a=100 \mathrm{~nm}, \varepsilon_{\mathrm{rp}}=2\right)$ in a $10^{-3} \mathrm{~mol} / \mathrm{L} \mathrm{KCl}$ solution $\left(\varepsilon_{\mathrm{rs}}=78.5\right)$, and with negligible ionic conduction in the stagnant layer. In this figure, we plot the variation of the real and imaginary parts of $\delta \varepsilon_{\mathrm{r}}^{*}$ with frequency. Note the very significant effect of the double-layer polarization on the low-frequency dielectric constant of a suspension. The variation with frequency is also noticeable, and both the $\alpha$ - (around $\left.10^{5} \mathrm{~s}^{-1}\right)$ and Maxwell-Wagner $\left(\sim 2 \times 10^{7} \mathrm{~s}^{-1}\right)$ relaxations are observed, the amplitude of the latter being much smaller than that of the former for the conditions chosen. No other electrokinetic technique can provide such a clear account of the double-layer relaxation processes. The effect of $\zeta$-potential on the frequency dependence of the dielectric constant is plotted in Fig. 6: the dielectric increment always increases with $\zeta$, as a consequence of the larger concentration of counterions in the EDL: all processes responsible for LFDD are amplified for this reason.

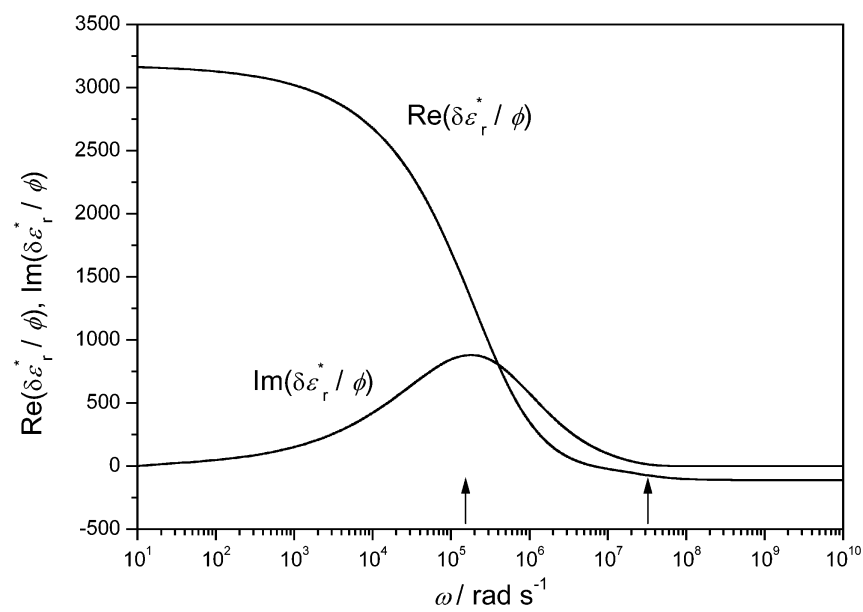

Fig. 5 Real and imaginary parts of the dielectric increment $\delta \varepsilon_{\mathrm{r}}^{*}$ (divided by the volume fraction $\phi$ ) for dilute suspensions of 100-nm particles in $\mathrm{KCl}$ solution, as a function of the frequency of the applied field for $\kappa a=10$ and $\zeta=100 \mathrm{mV}$. The arrows indicate the approximate location of the $\alpha$ (low frequency) and Maxwell-Wagner-O'Konski relaxations. 


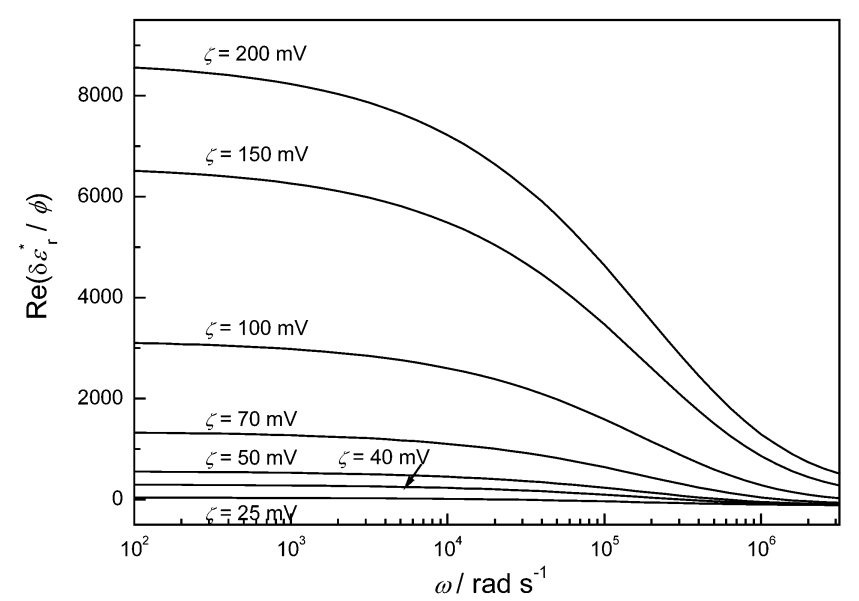

Fig. 6 Real part of the dielectric increment $\delta \varepsilon_{\mathrm{r}}^{*}$ (per volume fraction) as a function of the frequency of the applied field for dilute suspensions of $100-\mathrm{nm}$ particles in $\mathrm{KCl}$ solution. The $\zeta$-potentials are indicated, and in all cases, $\kappa a=10$.

The procedure for obtaining $\zeta$ from LFDD measurements is somewhat involved. It is advisable to determine experimentally the dielectric constant (or, equivalently, the conductivity) of the suspension over a wide frequency range, and use eqs. I.2 and I.3 (see Appendix I) to estimate the LFDD curve that best fits the data. A simpler routine is to measure only the low-frequency values, and deduce $\zeta$ using the same equations, but substituting $\omega=0$. However, the main experimental problems occur at low frequencies (see Section 3.4.3).

c. Dilute suspensions of nonconducting spherical particles with arbitrary $\kappa a$, and negligible $K^{\sigma \mathrm{i}}$

In this situation, there are no analytical expressions relating LFDD measurements to $\zeta$. Instead, numerical calculations based on DeLacey and White's treatment [66] are recommended. As before, the computing routine should be constructed to perform the calculations a number of times with different $\zeta$-potentials as inputs, until agreement between theory and experiment is obtained over a wide frequency range (or, at least, at low frequencies).

d. Dilute suspensions of nonconducting spherical particles with $\kappa a \gg>1$ and SLC

The problem of generalizing the theory by taking into account surface conduction caused by ions situated in the hydrodynamically stagnant layer has been dealt with in $[60,61,64]$. In theoretical treatments, SLC is equated to conduction within the Stern layer.

According to these models, the dielectric dispersion is determined by both $\zeta$ and $K^{\sigma \mathrm{i}}$. This means that, as discussed before, additional information on $K^{\sigma i}$ (see the methods described in Section 4.4) must accompany the dielectric dispersion measurements. Using dielectric dispersion data alone can only yield information about the total surface conductivity [66].

e. Dilute suspensions of nonconducting spherical particles with arbitrary $\kappa a$ and SLC.

Only numerical methods are available if this is the physical nature of the system under study. The reader is referred to [13-16,61,62,67-69]. Figure 7 illustrates how important the effect of SLC on $\operatorname{Re}\left[\varepsilon_{\mathrm{r}}^{*}(\omega)\right]$ can be for the same conditions as in Fig. 5. Roughly, the possibility of increased ionic mobilities in the stagnant layer brings about a systematically larger dielectric increment of the suspension: surface currents are larger for a conducting stagnant layer, and hence the electrolyte concentration gradients, ultimately responsible for the dielectric dispersion, will also be increased. 


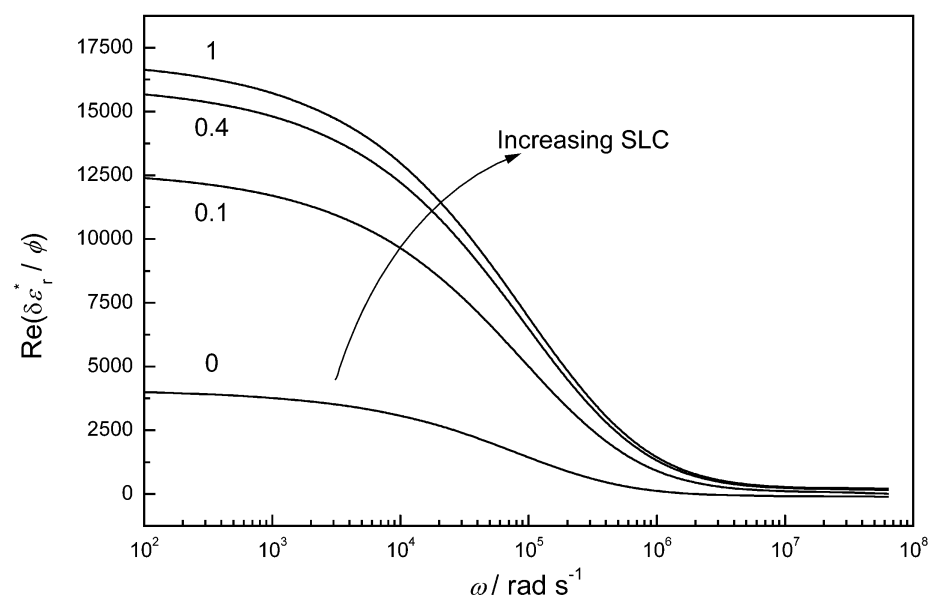

Fig. 7 Real part of the dielectric increment (per volume fraction) of dilute suspensions as in Fig. 5. The curves correspond to increasing importance of SLC; the ratios between the diffusion coefficients of counterions in the stagnant layer and in the bulk electrolyte are indicated (the lower curve corresponds to zero SLC).

f. Nondilute suspensions of nonconducting spherical particles with large $\kappa a$ and negligible $K^{\sigma \mathrm{i}}$

Considering that many suspensions of technological interest are rather concentrated, the possibilities of LFDD in the characterization of moderately concentrated suspensions have also received attention. This requires establishing a theoretical basis relating the dielectric or conductivity increments of such systems to the concentration of particles [70-72]. Here, we focus on a simplified model [73] that allows the calculation of the volume-fraction dependence of both the low-frequency value of the real part of $\delta \varepsilon_{\mathrm{r}}^{*}$, and of the characteristic time $\tau_{\alpha}$ of the $\alpha$-relaxation. The starting point is the assumption that $L_{\mathrm{D}}$, the length scale over which ionic diffusion takes place around the particle, can be averaged in the following way between the values of a very dilute $\left(L_{\mathrm{D}} \approx a\right)$ and a very concentrated $\left(L_{\mathrm{D}} \approx b-a ; b\right.$ is half the average distance between the centers of neighboring particles) dispersion:

$$
L_{\mathrm{D}}=\left(\frac{1}{a^{2}}+\frac{1}{(b-a)^{2}}\right)^{-1 / 2}
$$

or, in terms of the particle volume fraction

$$
L_{\mathrm{D}}=a\left(1+\frac{1}{\left(\phi^{-1 / 3}-1\right)^{2}}\right)^{-1 / 2}
$$

From these expressions, the simplified model allows us to obtain the dielectric increment at low frequency as follows. Let us call

$$
\Delta \varepsilon(0) \equiv \frac{\operatorname{Re}\left[\delta \varepsilon_{\mathrm{r}}^{*}(0)\right]}{\phi}
$$

the specific (i.e., per unit volume fraction) dielectric increment (for $\omega \rightarrow 0$ ). In the case of dilute suspensions, this quantity is a constant (independent of $\phi$ ), that we denote $\Delta \varepsilon_{\mathrm{d}}(0)$ : 


$$
\left.\Delta \varepsilon_{\mathrm{d}}(0) \equiv \frac{\operatorname{Re}\left[\delta \varepsilon_{\mathrm{r}}^{*}(0)\right]}{\phi}\right|_{\phi \rightarrow 0}
$$

The model allows us to relate eq. 69 with eq. 70 through the volume-fraction dependence of $L_{\mathrm{D}}$ :

$$
\Delta \varepsilon(0)=\Delta \varepsilon_{\mathrm{d}}(0)\left(1+\frac{1}{\left(\phi^{-1 / 3}-1\right)^{2}}\right)^{-3 / 2}
$$

A similar relationship can be established between dilute and concentrated suspensions in the case of the relaxation frequency $\omega_{\alpha}=1 / \tau_{\alpha}$ :

$$
\omega_{\alpha}=\omega_{\alpha d}\left(1+\frac{1}{\left(\phi^{-1 / 3}-1\right)^{2}}\right)
$$

where $\omega_{\alpha d}$ is the reciprocal of the relaxation time for a dilute suspension. Using this model, the dielectric increment and characteristic frequency of a concentrated suspension can be related to those corresponding to the dilute case which, in turn, can be related, as discussed above, to the $\zeta$-potential and other double-layer parameters. A general treatment of the problem, valid for arbitrary values of $\kappa a$ and $\zeta$ can be found in [74].

Summing up, we can say that the dielectric dispersion of suspensions is an interesting physical phenomenon, extremely sensitive to the characteristics of the particles, the solution, and their interface. It can provide invaluable information on the dynamics of the EDL and the processes through which it is altered by the application of an external field. Because of the experimental difficulties involved in its determination, it is unlikely that dielectric dispersion measurements alone can be useful as a tool to obtain the $\zeta$-potential of the dispersed particles.

\subsubsection{Experimental techniques available}

One of the most usual techniques for measuring the dielectric permittivity and/or the conductivity of suspensions as a function of the frequency of the applied field, is based on the use of a conductivity cell connected to an impedance analyzer. This technique has been widely employed since it was first proposed by Fricke and Curtis [75]. In most modern set-ups, the distance between electrodes can be changed (see, e.g., [69,76-79]). The need for variable electrode separation stems from the problem of electrode polarization at low frequencies, since at sufficiently low frequencies the electrode impedance dominates over that of the sample. The method makes use of the assumption that electrode polarization does not depend on their distance. A so-called quadrupole method has been recently introduced [80] in which the correction for electrode polarization is optimally carried out by proper calibration. Furthermore, the method based on the evaluation of the logarithmic derivative of the imaginary part of raw $\varepsilon^{*}(\omega)$ data also seems to be promising [81].

These are not, however, the only possible procedures. A four-electrode method has also been employed with success $[60,61,68]$ in this case, since the sensing and current-supplying electrodes are different, polarization is not the main problem, but the electronics of the experimental set-up is rather complicated.

\subsubsection{Samples for LFDD measurements}

There are no particular restrictions to the kind of colloidal particles that can be studied with the LFDD technique. The obvious precautions involve avoiding sedimentation of the particles during measurement, and control of the stability of the suspensions. LFDD quantities are most sensitive to particle size, particle concentration, and temperature. Hence, the constancy of the latter is essential. Another impor- 
tant concern deals with the effect of electrode polarization. Particularly at low frequencies, electrode polarization can be substantial and completely invalidate the data. This fact imposes severe limitations on the electrolyte concentrations that can be studied; it is very hard to obtain data for ionic strengths in excess of 1 to $5 \mathrm{mmol} \mathrm{L}^{-1}$.

\subsection{Electroacoustics}

\subsubsection{Operational definitions; recommended symbols and terminology; experimentally available quantities}

Terminology

The term "electroacoustics" refers to two kinds of closely related phenomena:

- $\quad$ Colloid vibration current $\left(I_{\mathrm{CV}}\right)$ and colloid vibration potential $\left(U_{\mathrm{CV}}\right)$ are two phenomena in which a sound wave is passed through a colloidal dispersion and, as a result, electrical currents and fields arise in the suspension. When the wave travels through a dispersion of particles whose density differs from that of the surrounding medium, inertial forces induced by the vibration of the suspension give rise to a motion of the charged particles relative to the liquid, causing an alternating electromotive force. The manifestations of this electromotive force may be measured in a way depending on the relation between the impedance of the suspension and the properties of the measuring instrument, either as $I_{\mathrm{CV}}$ (for small impedance of the meter) or as $U_{\mathrm{CV}}$ (for large one).

- The reciprocal effect of the above two phenomena is the electrokinetic sonic amplitude (ESA), in which an alternating electric field is applied to a suspension and a sound wave arises as a result of the motion of the particles caused by their ac electrophoresis.

Colloid vibration potential/current may be considered as the ac analog of sedimentation potential/current. Similarly, ESA may be considered as the ac analog of classical electrophoresis. The relationships between electroacoustics and classical EKP may be used for testing modern electroacoustic theories, which should at least provide the correct limiting transitions to the well-known and well-established results of the classical electrokinetic theory. A very important advantage of the electroacoustic techniques is the possibility they offer to be applied to concentrated dispersions.

Experimentally available quantities

Colloid vibration potential $\left(U_{C V}\right)$

If a standing sound wave is established in a suspension, a voltage difference can be measured between two different points in the standing wave. If measured at zero current flow, it is referred to as colloid vibration potential. The measured voltage is due to the motion of the particles: it alternates at the frequency of the sound wave and is proportional to another measured value, $\Delta P$, which is the pressure difference between the two points of the wave. The kinetic coefficient, equal to the ratio

$$
\frac{U_{\mathrm{CV}}}{\Delta p}=\frac{U_{\mathrm{CV}}}{\Delta p}\left(\omega, \phi, \Delta \rho / \rho, K^{\sigma \mathrm{i}}, \zeta, \eta, a\right)
$$

characterizes the suspension. In eq. $73, \Delta \rho$ is the difference in density between the particles and the suspending fluid, of density $\rho$.

Colloid vibration current $\left(I_{C V}\right)$

If the measurements of the electric signal caused by the sound wave in the suspension, are carried out under zero $U_{\mathrm{CV}}$ conditions (short-circuited), an ac $I_{\mathrm{CV}}$ can be measured. Its value, measured between two different points in the standing wave, is also proportional to the pressure difference between those 
two points, and the kinetic coefficient $I_{\mathrm{CV}} / \Delta p$ characterizes the suspension and is closely related to $U_{\mathrm{CV}} / \Delta p:$

$$
\frac{I_{\mathrm{CV}}}{\Delta p}=\frac{I_{\mathrm{CV}}}{\Delta p}\left(\omega, \phi, \Delta \rho / \rho, K^{\sigma \mathrm{i}}, \zeta, \eta, a\right)=K^{*} \frac{U_{\mathrm{CV}}}{\Delta p}
$$

\section{Electrokinetic sonic amplitude (ESA)}

This refers to the measurement of the sound wave amplitude, which is caused by the application of an alternating electric field to a suspension of particles of which the density is different from that of the suspending medium. The ESA signal (i.e., the amplitude $A_{\mathrm{ESA}}$ of the sound pressure wave generated by the applied electric field) is proportional to the field strength $E$, and the kinetic coefficient $A_{\mathrm{ESA}} / E$ can be expressed as a function of the characteristics of the suspension

$$
\frac{A_{\mathrm{ESA}}}{E}=\frac{A_{\mathrm{ESA}}}{E}\left(\omega, \phi, \Delta \rho / \rho, K^{\sigma \mathrm{i}}, \zeta\right)
$$

Measurement of $I_{\mathrm{CV}}$ or $A_{\mathrm{ESA}}$ rather than $U_{\mathrm{CV}}$ has the operational advantage that it enables measurement of the kinetic characteristics, $I_{\mathrm{CV}} / \Delta p$ and $A_{\mathrm{ESA}} / E$, which are independent of the complex conductivity $K^{*}$ of the suspension, and thus knowledge of $K^{*}$ is not a prerequisite for the extraction of the $\zeta$-potential from the interpretation of the electroacoustic measurements. Note, however, that if SLC is significant, as with the other EKP, additional measurements will be needed, as both $\zeta$ and $K^{\sigma \mathrm{i}}$ are required to fully characterize the interface.

\subsubsection{Estimation of the $\zeta$-potential from $U_{C V}, I_{C V}$, or $A_{E S A}$}

There are two recent methods for the theoretical interpretation of the data of electroacoustic measurements and extracting from them a value for the $\zeta$-potential. One is based on the symmetry relation proposed in $[82,83]$ to express both kinds of electroacoustic phenomena (colloid vibration potential/current and ESA) in terms of the same quantity, namely the dynamic electrophoretic mobility, $u_{\mathrm{d}}^{*}$, which is the complex, frequency-dependent analog of the normal direct current (dc) electrophoretic mobility. The second method is based on the direct evaluation of $I_{\mathrm{CV}}$ without using the symmetry relations, and hence it is not necessarily based on the concept of dynamic electrophoretic mobility. Both methods for $\zeta$-potential determination from electroacoustic measurements are briefly described below.

Using the dynamic mobility method has some advantages: (i) the zero frequency limiting value of $u_{\mathrm{d}}^{*}$ is the normal electrophoretic mobility, and (ii) the frequency dependence of $u_{\mathrm{d}}^{*}$ can be used to estimate not only the $\zeta$-potential, but also (for particle radius $>-40 \mathrm{~nm}$ ) the particle size distribution. Since the calculation of the $\zeta$-potential in the general case requires a knowledge of $\kappa a$, it is helpful to have available the most appropriate estimate of the average particle size (most colloidal dispersions are polydisperse, and there are many possible "average" sizes which might be chosen). Although the calculation of the $\zeta$-potential from the experimental measurements would be a rather laborious procedure, the necessary software for effecting the conversion is provided as an integral part of the available measuring systems, for both dilute and moderately concentrated sols. The effects of SLC can also be eliminated in some cases, without access to alternative measuring devices, simply by undertaking a titration with an indifferent electrolyte.

In the case of methods based on the direct evaluation of $I_{\mathrm{CV}}$, the use of different frequencies, if available, or of acoustic attenuation measurements, allows the determination of particle size distributions.

Method based on the concept of dynamic electrophoretic mobility

The symmetry relations lead to the following expressions, relating the different electroacoustic phenomena to the dynamic electrophoretic mobility, $u_{\mathrm{d}}^{*}[82,83]$ : 


$$
\begin{aligned}
& \frac{U_{\mathrm{CV}}}{\Delta p} \propto \phi \frac{\Delta \rho}{\rho} \frac{u_{\mathrm{d}}^{*}}{K^{*}} \\
& \frac{I_{\mathrm{CV}}}{\Delta p} \propto \phi \frac{\Delta \rho}{\rho} u_{\mathrm{d}}^{*} \\
& \frac{A_{\mathrm{ESA}}}{E} \propto \phi \frac{\Delta \rho}{\rho} u_{\mathrm{d}}^{*}
\end{aligned}
$$

Although the kinetic coefficients on the right-hand side of these relations (both magnitude and phase) can readily be measured at any particle concentration, there is some difficulty (see below) in the conversion of $u_{\mathrm{d}}^{*}$ to a $\zeta$-potential, except for the simplest case of spherical particles in fairly dilute suspensions (up to a few vol \%). In this respect, the situation is similar to that for the more conventional electrophoretic procedures. There are, though, some offsetting advantages. In particular, the ability to operate on concentrated systems obviates the problems of contamination which beset some other procedures. It also makes possible meaningful measurements on real systems without the need for extensive dilution, which can compromise the estimation of $\zeta$-potential, especially in emulsions systems.

Dilute systems (up to $\sim 4$ vol \%)

1. For a dilute suspension of spherical particles with $\kappa a \gg>1$ and arbitrary $\zeta$-potential, the following equation can be used [84], which relates the dynamic mobility with the $\zeta$-potential and other particle properties:

$$
u_{\mathrm{d}}^{*}=\left(\frac{2 \varepsilon_{\mathrm{rs}} \varepsilon_{0} \zeta}{3 \eta}\right)(1+f) G(\alpha)
$$

The restriction concerning double-layer thickness requires in practice that $\kappa a>\sim 20$ for reliable results, although the error is usually tolerable down to about $\kappa a=13$.

The function $f$ is a measure of the tangential electric field around the particle surface. It is a complex quantity, given by

$$
f=\frac{1+i \omega^{\prime}-\left[2 D u+i \omega^{\prime}\left(\varepsilon_{\mathrm{rp}} / \varepsilon_{\mathrm{rs}}\right)\right]}{2\left(1+i \omega^{\prime}\right)+\left[2 D u+i \omega^{\prime}\left(\varepsilon_{\mathrm{rp}} / \varepsilon_{\mathrm{rs}}\right)\right]}
$$

where $\omega^{\prime} \equiv \omega \varepsilon_{\mathrm{rs}} \varepsilon_{0} / K_{\mathrm{L}}$ is the ratio of the measurement frequency, $\omega$, to the Maxwell-Wagner relaxation frequency of the electrolyte. If it can be assumed that the tangential current around the particle is carried essentially by ions outside the shear surface, then $D u$ is given by the $D u^{\mathrm{d}}$ (eqs. 16-19); see also [85]*.

The function $G(\alpha)$ is also complex and given by

$$
G(\alpha)=\frac{1+(1+i) \sqrt{\alpha / 2}}{1+(1+i) \sqrt{\alpha / 2}+i \frac{\alpha}{9}(3+2 \Delta \rho / \rho)}
$$

\footnotetext{
*If this assumption breaks down, $D u$ must be estimated by reference to a suitable surface conductance model or, preferably, by direct measurement of the conductivity over the frequency range involved in the measurement (normally from about 1 to $40 \mathrm{MHz}$ ) [86]. Another procedure involved the analysis of the results of a salt titration (see previous subsection).
} 
It is a direct measure of the inertia effect. The dimensionless parameter $\alpha$ is defined as

$$
\alpha=\frac{\omega a^{2} \rho}{\eta}
$$

so $G$ is strongly dependent on the particle size. $G$ varies monotonically from a value of unity, with zero phase angle, when $a$ is small (less than $0.1 \mu \mathrm{m}$ typically) to a minimum of zero and a phase lag of $\pi / 4$ when $a$ is large (say, $a>10 \mu \mathrm{m}$ ).

Equations 80-82 are, as mentioned above, applicable to systems of arbitrary $\zeta$-potential, even when conduction occurs in the stagnant layer, in which case $D u$ in eq. 80 must be properly evaluated. They have been amply confirmed by measurements on model suspensions [86], and have proved to be of particular value in the estimation of high $\zeta$-potentials [87]. The maximum that appears in the plot of dc mobility against $\zeta$-potential ([22], see also Fig. 2) gives rise to an ambiguity in the assignment of the $\zeta$-potential, which does not occur when the full dynamic mobility spectrum is available.

2. For double layers with $\kappa a<\sim 20$, there are approximate analytical expressions $[88,89]$ for dilute suspensions of spheres, but they are valid only for low $\zeta$-potentials. They have been checked against the numerical solutions for general $\kappa a$ and $\zeta[90,91]$, and those numerical calculations have been subjected to an experimental check in [92].

3. The effect of particle shape has been studied in [93,94], again in the limit of dilute systems with thin double layers. This analysis has been extended in [95] to derive formulae for cylindrical particles with zero permittivity and low $\zeta$-potentials, but for arbitrary $\kappa a$. The results are consistent with those of $[93,94]$.

Concentrated systems

The problem of considering the effect on the electroacoustic signal of hydrodynamic or electrostatic interactions between particles in concentrated suspensions was first theoretically tackled [96] by using the cell model of Levine and Neale $[97,98]$ to provide a solution that was claimed to be valid for $U_{\mathrm{CV}}$ measurements on concentrated systems (see [87] for a discussion on the validity of such approach in the high-frequency range).

It is possible to deal with concentrated systems at high frequency without using cell models in the case of near neutrally buoyant systems (where the relative density compared to water is in the range $0.9-1.5)$ using a procedure developed by O'Brien $[99,100]$. In that case, the interparticle interactions can be treated as pairwise additive and only nearest-neighbor interactions need to be taken into account. An alternative approach is to estimate the effects of particle concentration considering in detail the behavior of a pair of particles in all possible orientations [101,102]. Empirical relations have been developed that appear to represent the interaction effects for more concentrated suspensions up to volume fractions of $30 \%$ at least. In [103], an example can be found where the dynamic mobility was analyzed assuming the system to be dilute. The resulting value of the $\zeta$-potential $\left(\zeta_{\mathrm{app}}\right)$ was then corrected for concentration using the semi-empirical relation:

$$
\zeta_{\text {corr }}=\zeta_{\text {app }} \exp \{2 \phi[1+s(\phi)]\}, \quad \text { with } s(\phi)=\frac{1}{1+(0.1 / \phi)^{4}}
$$

Finally, O'Brien et al. [104] have recently developed an effective analytical solution to the concentration problem for the dynamic mobility.

Method based on the direct evaluation of the $I_{\mathrm{CV}}$

This approach [105] applies a "coupled phase model" [106-109] for describing the speed of the particle relative to the liquid. The Kuwabara cell model [110] yields the required hydrodynamic parameters, 
such as the drag coefficient, whereas the Shilov-Zharkikh cell model [111] was used for the generalization of the Kuwabara cell model to the electrokinetic part of the problem.

The method allows the study of polydisperse systems without using any superposition assumption. It is important in concentrated systems, where superposition does not work because of the interactions between particles.

An independent exact expression for $I_{\mathrm{CV}}$ in the quasi-stationary case of low frequency, using Onsager's relationship and the HS equation, and neglecting the surface conductivity effect $(D u<<1)$, is [105]

$$
\left.I_{\mathrm{CV}}\right|_{\omega \rightarrow 0}=\frac{\varepsilon_{\mathrm{rs}} \varepsilon_{0} \zeta K_{\mathrm{DC}} \phi}{\eta K_{\mathrm{L}}} \frac{\left(\rho_{\mathrm{p}}-\rho_{\mathrm{s}}\right)}{\rho_{\mathrm{s}}} \frac{\mathrm{d} p}{\mathrm{~d} x}
$$

where $x$ is the coordinate in the direction of propagation of the pressure wave, $\rho_{\mathrm{s}}$ is the density of the suspension (not of the dispersion medium: this is essential for concentrated suspensions, see [112]). Equation 84 is the analog of the HS equation for stationary electrophoretic mobility. It has been claimed to be valid over the same wide range of real dispersions, with particles of any shape and size, and any concentration.

\subsubsection{Experimental procedures}

In the basic experimental procedures, an ac voltage is applied to a transducer that produces a sound wave which travels down a delay line and passes into the suspension. This acoustic excitation causes a very small periodic displacement of the fluid. Although the particles tend to stay at rest because of their inertia, the ionic cloud surrounding them tends to move with the fluid to create a small oscillating dipole moment. The dipole moment from each particle adds up to create an electric field that can be sensed by the receiving transducer. The voltage difference that then appears between the electrodes (measured at zero current flow) is proportional to $U_{\mathrm{CV}}$. The current, measured under short-circuit conditions, is a measure of $I_{\mathrm{CV}}$. Alternatively, an ac electric field can be applied across the electrodes. A sound wave is thereby generated near the electrodes in the suspension, and this wave travels along the delay line to the transducer. The transducer output is then a measure of the ESA effect. For both techniques, measurements can be performed for just one frequency or for a set of frequencies ranging between 1 and $100 \mathrm{MHz}$, depending on the instrument.

Recently, the term electroacoustic spectrometry has been coined to refer to the measurement of the dynamic mobility as a function of frequency (see Fig. 8 for some examples of the kind of spectra that can be obtained). The plot of magnitude and phase of $u_{\mathrm{d}}^{*}$ over a range of (MHz) frequencies may yield information about the particle size as well as the $\zeta$-potential [86,113], provided the particles are in a suitable size range (roughly $0.05 \mu \mathrm{m}<a<5 \mu \mathrm{m}$ ). For smaller particles, the signal provides a measure of the $\zeta$-potential, but an independent estimate of size is needed for quantitative results. That can be provided by, for instance, ultrasonic attenuation measurements with the same device.

\subsubsection{Samples for calibration}

The original validation of the ESA technique was achieved by comparing the theoretical dynamic mobility spectrum, as given by eqs. $80-82$, with the experimentally measured values. That comparison has been done for a number of silica, alumina, titania, and goethite samples, and some pharmaceutical emulsion samples [86]. Presently, the calibration standard is a salt solution (potassium dodecatungstosilicate). This salt has a significant ESA signal that can be calculated from its known transport and equilibrium thermodynamic properties (namely, the transport numbers and partial molar volumes of the ions) [85]. Once calibrated, the instrument allows a direct measure of both the size and $\zeta$-potential of the particles. When applied to monodisperse suspensions of spherical particles, the instrument gives results that agree, for both size and $\zeta$-potential, with more conventional methods. 

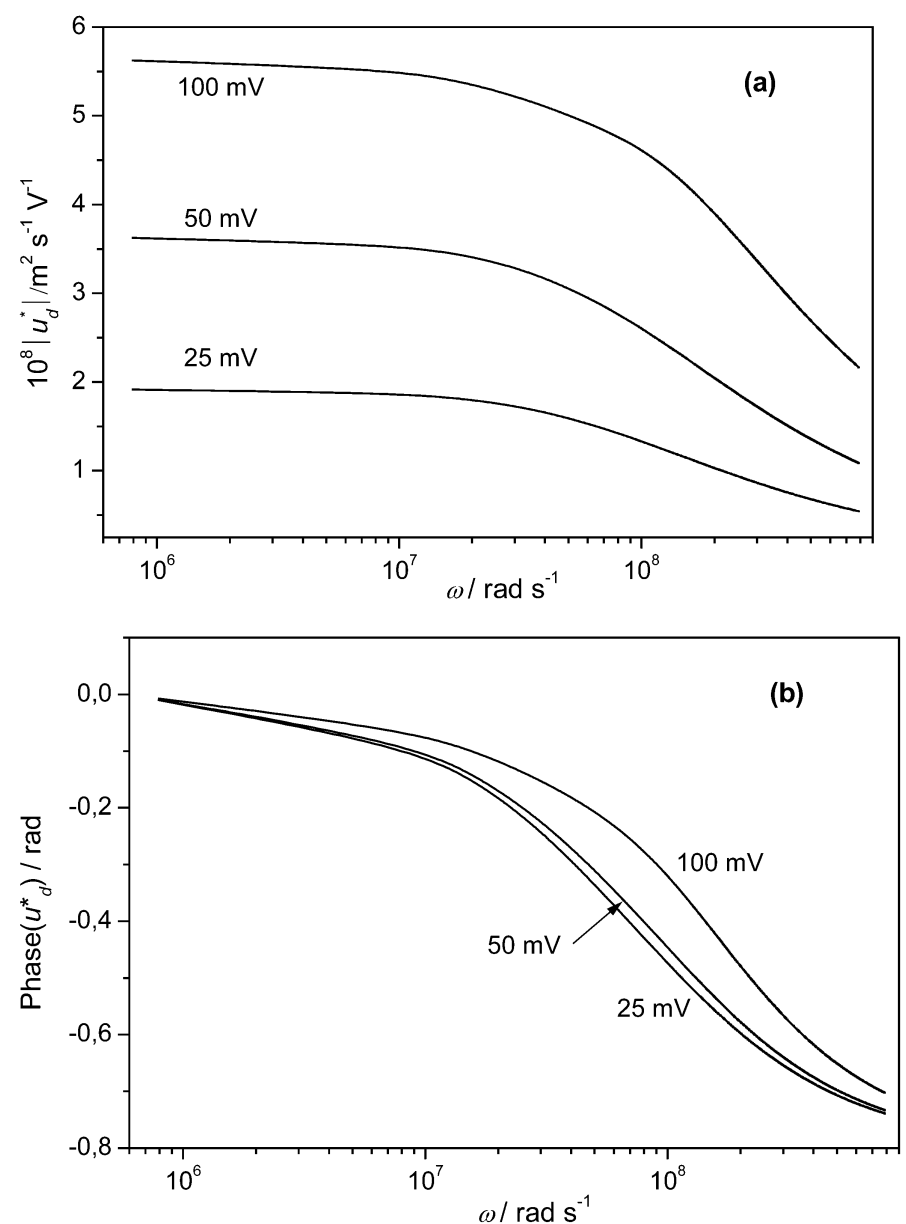

Fig. 8 Modulus (a) and phase angle (b) of the dynamic mobility of spherical particles in a $\mathrm{KCl}$ solution with $\kappa a=$ 20 as a function of frequency for different $\zeta$-potentials; cf., eqs. 80-82. Parameters: particle radius $100 \mathrm{~nm}$; dielectric constant of the particles (dispersion medium): 2 (78.54); density of the particles (dispersion medium): $5 \times 10^{3}\left(1 \times 10^{3}\right) \mathrm{kg} \mathrm{m}^{-3}$.

In $I_{\mathrm{CV}}$ measurements, calibration is often carried out using a colloid with known $\zeta$-potential, such as Ludox TM (commercially available colloidal silica), which is diluted to a mass concentration of $0.1 \mathrm{~g} / \mathrm{cm}^{3}$ with $10^{-2} \mathrm{~mol} \mathrm{~L}^{-1} \mathrm{KCl}$.

\section{ELECTROKINETICS IN NONAQUEOUS SYSTEMS}

\subsection{Difference with aqueous systems: Permittivity}

The majority of the investigations of EKP are devoted to aqueous systems, for which the main charging mechanisms of solid/water interfaces have been established and the EDL properties are fairly well known. All general theories of EKP assume that the equations relating them to the $\zeta$-potential are applicable to any liquid medium characterized by two bulk properties: electric permittivity $\varepsilon_{\mathrm{rs}} \varepsilon_{0}$ and viscosity $\eta$. The value of $\varepsilon_{\mathrm{rs}}$ is an important parameter of the liquid phase, because it determines the dissociation of the electrolytes embedded in it. Most nonaqueous solvents are less polar than water, and 
hence $\varepsilon_{\mathrm{rS}}$ is lower. All liquids can be classified roughly as nonpolar $\left(\varepsilon_{\mathrm{rS}} \leq 5\right)$, weakly polar $\left(5<\varepsilon_{\mathrm{rs}} \leq\right.$ $12)$, moderately polar $\left(12<\varepsilon_{\mathrm{rs}} \leq 40\right)$, and polar $\left(\varepsilon_{\mathrm{rs}}>40\right)$.

For $\varepsilon_{\mathrm{rs}}>40$, dissociation of most dissolved electrolytes is complete, and all equations concerning EDL or EKP remain unmodified, except that a lower value for $\varepsilon_{\mathrm{rs}}$ has to be used. For moderately polar solvents, the electrolyte dissociation is incomplete, which means that the concentration of charged species (i.e., the species that play a role in EDL or EKP) may be lower than the corresponding electrolyte concentration. Moreover, the charge number of the charge carriers can become lower. For instance, in solutions of $\mathrm{Ba}\left(\mathrm{NO}_{3}\right)_{2}$, we may find not (only) $\mathrm{Ba}^{2+}$ and $\mathrm{NO}_{3}^{-}$ions, but (also) $\mathrm{Ba}\left(\mathrm{NO}_{3}\right)^{+}$complexes. The category of weakly polar solvents is a transition to the class of nonpolar liquids, for which the relative permittivity (at infinite frequency) equals the square of the index of refraction. Such liquids exhibit no self-dissociation, and the notion of electrolytes almost completely dissociated loses its meaning. However, even in such media, special types of molecules may dissociate to some extent and give rise to the formation of EDL and EKP. It is this group that we shall now emphasize.

Unlike in aqueous media, dissociation in these solvents occurs only for potential electrolytes that contain ions of widely different sizes. Once dissociation occurs, the tendency for re-dissociation is relatively small because the charge on the larger ion is distributed over a large area. However, the concentration of ions $\left(c_{+}, c_{-}\right)$is very small. An indication of the magnitude of $c_{+}$and $c_{-}$can be obtained from conductivity measurements.

Particles embedded in such a liquid can become charged when one type of ion can be preferentially adsorbed. Typically, the resulting surface charges, $\sigma^{0}$, are orders of magnitude lower than in aqueous systems. However, because of the very low EDL capacitance, the resulting surface potentials, $\psi^{0}$, are of the same order of magnitude as in aqueous systems.

The very slow decay of the potential with distance has two consequences. First, as the decay between surface and slip plane is negligible, $\psi^{0} \approx \zeta$. This simplifies the analysis. Second, the slow decay implies that colloid particles "feel" each other's presence at long range. So, even dilute sols may behave physically as "concentrated" and formation of small clusters of coagulated particles is typical.

Interestingly, electrophoresis can help in making a distinction between concentrated and dilute systems by studying the dependence of the electrophoretic mobility on the concentration of dispersed particles. If there is no dependence, the behavior is that of a dilute system. In this case, equations devised for dilute systems can be used. Otherwise, the behavior is effectively that of a concentrated system, and ESA or $U_{\mathrm{CV}}$ measurements are more appropriate [86].

\subsection{Experimental requirements of electrokinetic techniques}

The methods described are also applicable to electrokinetic measurements in nonaqueous systems, but some precautions need to be taken. Microelectrophoresis cells are often designed for aqueous and water-like media whose conductivity is high relative to that of the material from which the cell is made. A homogeneous electric field between the electrodes of such cells filled with well- or moderately conducting liquids is readily achieved. However, when the cell is filled with a low-conductivity liquid, the homogeneity of the electric field can be disturbed by the more conducting cell walls and/or by the surface conduction due to adsorption of traces of water dissolved from the solvents of low polarity on the more hydrophilic cell walls. Special precautions (coating the walls with hydrophobic layers) are necessary to improve the field characteristics. The electric field in cells of regular geometrical shape is calculated from the measured current density and the conductivity of the nonaqueous liquid. Because of the low ionic strength of the latter, electrode polarization may occur and it can sometimes be observed as bubble formation. Hence, an additional pair of electrodes is often used for the measurements of the voltage gradient across the cell.

For the correct measurement of the streaming potential in nonaqueous systems, attention must be paid to ensure that the resistance of the capillary, plug, or diaphragm filled with liquid is at least 100 
times less than the input impedance of the electrical measuring device. The usual practice is to use millivoltmeter-electrometers with input resistance higher than $10^{14} \Omega$ and platinum gauze electrodes placed in contact with the ends of the capillary or plug for both resistance and streaming potential measurements. The resistance is usually measured with an ac bridge in the case of polar solvents (typical frequencies used are around a few $\mathrm{kHz}$ ) and by dc methods in the case of nonpolar or weakly polar liquids. The use of data recording on the electrometer output is common practice to check the rate of attainment of equilibrium and possible polarization effects.

\subsection{Conversion of electrokinetic data into $\zeta$-potentials}

The first step in interpreting the electrokinetic behavior of nonaqueous systems must be to check whether the system behaves as a dilute or as a concentrated system (see Section 5.2). In the dilute regime, all theories described for aqueous systems can be used, provided one can find the right values of the essential parameters $\kappa a, K_{\mathrm{p}}$, and $K^{\sigma}$.

The calculation of $\kappa$ requires knowledge of the ionic strength of the solution; this, in turn, can be estimated from the measurement of the dialysate conductivity, $K_{\mathrm{L}}$, and knowledge of the mobilities and valences of the ionic species.

The effective conductivity of the solid particle, $K_{\text {pef }}$, including its surface conductivity* can be calculated from the experimental values of conductivities of the liquid $K_{\mathrm{L}}$, and of a dilute suspension of particles, $K$, with volume fraction $\phi$ using either Street's equation

$$
\frac{K_{\text {pef }}}{K^{\mathrm{L}}}=1+\frac{2}{3} \frac{\frac{K}{K_{\mathrm{L}}}-1}{\phi}
$$

or Dukhin's equation

$$
\frac{K_{\mathrm{pef}}}{K^{\mathrm{L}}}=2 \frac{(1-\phi)-\frac{K}{K_{\mathrm{L}}}(1+\phi / 2)}{(1-\phi) \frac{K}{K_{\mathrm{L}}}-(1+2 \phi)}
$$

which accounts for interfacial polarization.

For the estimation of the $\zeta$-potential in the case of electrophoresis, considering that low $\kappa a$ values are not rare, it is suggested to use Henry's theory, after substitution of $K_{\text {pef }}$ estimated as described above, for the particle conductivity. In formulas, it is suggested to employ eqs. 29 and 30.

Concerning streaming potential/current or electro-osmotic flow measurements, all the abovementioned features of nonaqueous systems must be taken into account to obtain correct values of $\zeta$-potential from this sort of data in either single capillaries, porous plugs, or diaphragms. In view of the low $\kappa a$ values normally attained by nonaqueous systems, the Rice and Whitehead curvature corrections are recommended [50]; see eqs. 37-39, 44, and 50.

Finally, electroacoustic characterization of $\zeta$-potential in nonaqueous suspensions requires subtraction of the background arising from the equilibrium dispersion medium. This is imperative because

\footnotetext{
*The low conductivity of nonaqueous media with respect to aqueous solutions is the main reason why often the finite bulk conductivity of the dielectric solids cannot be neglected. In contrast, if one can assume that no water is adsorbed at the interface, any SLC effect can be neglected. Furthermore, the joint adsorption of the ionic species of both signs or (and) the adsorption of such polar species as water at the solid/liquid interface can produce an abnormally high surface conduction. This is not due to the excess of free charges in the EDL, commonly taken into account in the parameter $K^{\sigma}$ of the generalized theories of EKP. Rather, it is conditioned by the presence of thin, highly conducting adsorption layers. Therefore, the ratio $K_{\text {pef }} / K_{\mathrm{L}}$ is an important parameter that has to be estimated for nonaqueous systems.
} 
the electroacoustic signal generated by particles in low- or nonpolar media is very weak. It is recommended to make measurements at several volume fractions in order to ensure that the signal comes, in fact, from the particles.

Note that there is one more issue that complicates the formulation of a rigorous electrokinetic theory in concentrated nonaqueous systems, namely, the conductivity of a dispersion of particles in such media becomes position-dependent, as the double layers of the particles may occupy most of the volume of the suspension. This is a significant theoretical obstacle in the elaboration of the theory. In the case of EKP based on the application of nonstationary fields (dielectric dispersion, electroacoustics), this problem can be overcome. This is possible because one of the peculiarities of low- and nonpolar liquids compared to aqueous systems is the very low value of the Maxwell-Wagner-O'Konski frequency, eqs. 57 and 58. This means that in the modern electroacoustic methods based on the application of electric or ultrasound fields in the $\mathrm{MHz}$ frequency region, all effects related to conductivity can be neglected, because that frequency range is well above the Maxwell-Wagner characteristic frequency of the liquid. This makes electroacoustic techniques most suitable for the electrokinetic characterization of suspensions in nonaqueous media.

\section{REMARKS ON NON-IDEAL SURFACES}

\subsection{General comments}

The general theory of EKP described so far strictly applies to ideal, nonporous, and rigid surfaces or particles. By ideal, we mean smooth (down to the scale of molecular diameters) and chemically homogeneous, and we use rigid to describe those particles and surfaces that do not deform under shear. We will briefly indicate interfaces that are nonporous and rigid as "hard" interfaces. For hard particles and surfaces, there is a sharp change of density in the transition between the particle or surface and the surrounding medium. Hard particles effectively lead to stacking of water (solvent, in general) molecules; that is, only for hard surfaces the notion of a stagnant layer close to the real surface is conceptually simple. Not many surfaces fulfill these conditions. For instance, polystyrenesulfonate latex colloid has, in fact, a heterogeneous interface: the hydrophilic sulfate end-groups of its polymer chains occupy some $10 \%$ of the total surface of the particles, the remainder being hydrophobic styrene. Moreover, the sulfate groups will protrude somewhat into the solution. Generally speaking, many interfaces are far from molecularly smooth, as they may contain pores or can be somewhat deformable. Such interfaces can briefly be indicated as "soft" interfaces. For soft interfaces, such as, for instance, rigid particles with "soft" or "hairy" polymer layers, gel-type penetrable particles and water-air or water-oil interfaces, the molecular densities vary gradually across the phase boundary. A main problem in such cases is the description of the hydrodynamics, and in some cases it is even questionable if a discrete slip plane can be defined operationally.

The difficulties encountered when interpreting experimental results obtained for non-ideal interfaces depend on the type and magnitude of the non-idealities and on the aim of the measurements. In practice, one can always measure some quantity (like $u_{\mathrm{e}}$ ), apply some equation (like HS) to compute what we can call an "effective" $\zeta$-potential, but the physical interpretation of such a $\zeta$-potential is ambiguous. We must keep in mind that the obtained value has only a relative meaning and should not be confused with an actual electrostatic potential close to the surface. Nevertheless, such an "effective $\zeta$ " can help us in practice, because it may give some feeling for the sign and magnitude of the part of the double-layer charge that controls the interaction between particles. When the purpose of the measurement is to obtain a realistic value of the $\zeta$-potential, there is no general recipe. It may be appropriate to use more than one electrokinetic method and to take into account the specific details of the non-ideality as well as possible in each model for the calculation of the $\zeta$-potential. If the $\zeta$-potentials resulting from both methods are similar, physical meaning can be assigned to this value. 
Below, we will discuss different forms of non-ideality in somewhat more detail. We will mainly point out what the difficulties are, how these can be dealt with and where relevant literature can be found.

\subsection{Hard surfaces}

Some typical examples of non-ideal particles that still can be considered as hard are discussed below. Attention is paid to size and shape effects, surface roughness, and surface heterogeneity. For hard nonideal surfaces, both the stagnant-layer concept and the $\zeta$-potential remain locally defined and experimental data provide some average electrokinetic quantity that will lead to an average $\zeta$-potential. The kind of averaging may depend on the electrokinetic method used, therefore, different methods may give different average $\zeta$-potentials.

\subsubsection{Size effects}

For rigid particles that are spherical with a homogeneous charge density, but differ in size, a rigorous value of the $\zeta$-potential can be found if the electrokinetic quantity measured is independent of the particle radius, $a$. In general, this will be the case for $\kappa a>>1$ (HS limit). In the case of electrophoresis, the mobility is also independent of $a$ for $\kappa a<1$ (HO limit). For other cases, the particle size will come into play and most of the time an average radius has to be used to calculate an average $\zeta$-potential [3,114]. The type of average radius used will, in principle, affect the average $\zeta$-potential. Furthermore, different EKP may require different averages. For instance, in [115] it was found that a simple number average is suitable for analysis of electrophoresis in polydisperse systems; a volume average, on the other hand, was found to be the best choice when discussing dielectric dispersion. In principle, the best solution would be found if the full size distribution is measured, but even in such a case the signal-processing procedure must be taken into account. For instance, in ELS methods, the scattering of light by particles of different sizes is determinant for the average $u_{\mathrm{e}}$ value found by the instrument. In this context, measuring the dynamic mobility spectrum may be useful in providing information on both the size and the $\zeta$-potential from the same signal.

\subsubsection{Shape effects}

For nonspherical particles (cylinders, discs, rods, etc.) of homogeneous $\zeta$-potential, the induced dipole moment is different for different orientations with respect to the applied field (i.e., it acquires a tensorial character). Only when the systems are sufficiently simple (cylinders, rods) is it sufficient to distinguish between a parallel and a normal orientation of the dipoles to the applied field [116]. In spite of these additional difficulties, some approximate approaches to either the electrophoresis [117-120] or the permittivity $[121,122]$ of suspensions of nonspherical particles are available.

A more complicated situation arises if the particles are polydisperse in shape and size. Except if $\kappa a \gg 1$ (HS equation valid) or if $\kappa a<<1$ (HO limit), the only (approximate) approach is to define an "equivalent spherical particle" (for instance, one having the same volume as the particle under study) and use theories developed for spheres. In that case, the "average" $\zeta$-potential that is obtained depends on the type(s) of polydispersity of the sample and the definition used for the "equivalent sphere".

\subsubsection{Surface roughness}

Most theories described so far assume that the interface is smooth down to a molecular scale. However, even the surfaces prepared with the strictest precautions show some degree of roughness, which will be characterized by $R$, the typical dimension of the mountains or valleys present on the surface. However, surface roughness (with $R$ as a measure of the roughness size) affects the position of the plane of shear except in conditions where $\kappa R>>1$ (HS limit) or $\kappa R<<1$ (roughness not seen). If it is assumed that the outer parts of the asperities determine the position of the slip plane, there will be diffusely bound or even free ions in the valleys. This will inevitably lead to large surface conductivities behind the apparent slip plane, and to an additional mechanism of stagnancy $[6,116,123]$. Due care must be taken to 
measure this conductivity and to take it into account in evaluating the $\zeta$-potentials. Experiments with surfaces of well-defined roughness are required to gain further insight in the complications.

We will not proceed with a more thorough description of these highly specialized — and mostly unsolved-topics. As a rule of thumb, we recommned that the reader deal with an interface of known, high roughness to use a simple approach based on the calculation of an effective $\zeta$-potential obtained under the assumption that the interface is smooth, but to refrain from using this effective $\zeta$ for further calculations. This is particularly true if the estimation of interaction energies between the particles is sought, as it has been shown [124] that asperities have a considerable effect on both electrostatic and van der Waals forces between colloid particles.

\subsubsection{Chemical surface heterogeneity}

In general, chemical surface heterogeneity will also lead to surface charge heterogeneity. When the charges do not protrude into the solution, the position of the slip plane is unaffected. With charge heterogeneity, often two extreme cases are considered: (1) a random or regular heterogeneity that is assumed to lead to one (smeared-out) surface charge density and also one (averaged) electrokinetic potential and electrokinetic charge density at given solution conditions; and (2) a patchwise heterogeneity with large patches. The patches will lead to an inhomogenous charge distribution. In this case, it is usually assumed that each patch has its own smeared-out charge density at given solution conditions. The characteristic size of the patches should be at least of the order of the Debye length, otherwise the surface may be considered as regularly heterogeneous with one smeared-out charge density over the entire surface. Particles with random heterogeneity can be treated in the same way as particles with a homogeneous charge density, so it does not present additional problems.

The patchwise heterogeneity case applies, for instance, to surfaces with different crystal faces. The electrokinetic theory for this type of surface has been considered by several authors $[118,119,125,126]$. Anderson et al. [127-129] have also performed experimental checks on the validity of some theoretical predictions. In the case of spherical particles, it has been demonstrated [130] that their motion depends on the first, second, and third moments of the $\zeta$-potential distribution along the surface of the particle. It is important to note that the second moment (dipole moment) brings about a rotational motion superimposed on the translational (when present) electrophoretic motion. Clay particles [126,131], with anionic charges at the plate surfaces and $\mathrm{pH}$-dependent charges at the edges, are typical examples of systems with patchwise heterogeneous surfaces.

A rather specific situation may appear in the case of sparse polyelectrolyte adsorption onto oppositely charged surfaces. This may lead to a mosaic-type charge distribution [132,133]. Not only the very concepts of slip plane and hence $\zeta$-potential are doubtful here, but also electrokinetic data alone can lead us to erroneous conclusions. For instance, one can find a high $\left|u_{\mathrm{e}}\right|$ value for particles with attached polyelectrolytes and from this predict a high colloidal stability. This might not be found, since the patchwise nature of the charges might induce flocculation even in systems that have considerable average $\zeta$-potential. Such instability will be due to attraction between oppositely charged patches (corresponding to regions with and without adsorbed polyelectrolyte).

\subsection{Soft particles}

Some familiar examples in which the interface must be considered as soft are discussed below. The examples refer to two different groups. The first consists of hard particles with hairy, grafted, or adsorbed layers and of particles that are (partially) penetrable. The hydrodynamic permeability and the conductivity in the permeable layer make the interpretation of the data complicated. The second group are the water-oil or water-air interfaces. Droplets and bubbles comprise a specific class of "soft" particles, for which the definition of the slip plane is an academic question. When and how this issue can be solved depends on the surface active components present at the interface. 


\subsubsection{Charged particles with a soft uncharged layer}

For a good understanding of charged hard particles covered by nonionic surfactants or uncharged polymers, the position of the slip plane and conduction behind the slip plane must be considered. It is very useful to also investigate the bare particles. Neutral adsorbed layers reduce the tangential fluid flow and the tangential electric current near a particle surface, but to a different extent [134]. Both reductions have to be considered for a correct interpretation of the electrokinetic results. By doing this, the comparison of the results between bare and covered particles may give information about the net particle potential and effective particle charge, the surface charge adjustment, and the adsorbed-layer thickness [135]. Let us mention that electroacoustic studies of the dynamic mobility spectrum of particles coated with adsorbed neutral polymer can give information on the thickness of the adsorbed layer and the shift of the slip plane due to the polymer [136].

\subsubsection{Uncharged particles with a soft charged layer}

The surface of many latex particles can be considered as being itself uncharged, but with charged groups at the end of oligomeric or polymeric "hairs" that protrude into the solution. The extension of the "hairs" from the surface into the liquid determines the position of the apparent slip plane. Due to the ion penetrability of the stagnant layer, the ionic strength will affect the distance between the surface and this (apparent) slip plane, and the electric conduction in the stagnant layer and this will lead to a more complex ionic strength dependence of the $\zeta$-potential than with rigid particles. Some studies account for these effects through the introduction of a "softness factor" [137,138] as a fitting parameter. Stein [139] discusses the electrokinetics of polystyrene latex particles in more detail. Hidalgo-Álvarez et al. [140] discuss the anomalous electrokinetic behavior of polymer colloids in general.

\subsubsection{Charged particles with a soft charged layer}

Charged particles with adsorbed or grafted polyelectrolyte or ionic surfactant layers fall in this class. The complications arising with these systems are similar to those mentioned for the uncharged surfaces with charged layers. Adsorbed layers impede tangential fluid flow; therefore, in the presence of the bound layer the hydrodynamic particle radius increases and the apparent slip plane is moved outwards. This affects the tangential electric current. In most cases, the particle surface and the bound layers will have opposite charges, and, therefore, the electrostatic potential profile is rather complicated. The EKP will be dominated by conduction within the slip plane and the potential at the hydrodynamic boundary relatively far away from the surface $[114,141,142]$. To unravel the situation, a systematic investigation is required that considers the electrokinetic behavior of both uncovered and covered particles, the shift of the apparent slip plane and the conduction behind the slip plane. When qualitative information is required with respect to the net charge of the particle plus adsorbed layer, the sign of the $\zeta$-potential is important, as it will indicate whether the particle charge is overcompensated or not (within the plane of shear).

\subsubsection{Ion-penetrable or partially penetrable particles}

Some proteins, many biological cells, and other natural particles are penetrable for water and ions. The most important complications for the description of the electrokinetic behavior of such particles are associated with the conductivity, the dielectric constant, and the liquid transport inside the particles. An additional complication occurs when the particles are able to swell depending on the solution conditions.

When the particles are only partially penetrable, we may consider them as hard with a gel-like corona. This situation is very similar to hard particles coated with a polyelectrolyte layer. In the limit of a very small particle with a very thick corona, the penetrable particle limit results. The simplest models assume that the electrical potential inside the gel layer is the Donnan potential, whereas the hindered motion of liquid in it is represented by a friction parameter incorporated in the Navier-Stokes equation [143-146]. Ohshima's theory of electrophoresis of soft spheres basically ranges from hard particles to penetrable particles. References to his work can be found in his review on EKP [140]. 
Systems with biological or medical relevance that have received some systematic attention are protein-coated latex particles [147], electrophoresis of biological cells [148], liposomes [149], and bacteria [150].

\subsubsection{Liquid droplets and gas bubbles in a liquid}

The electrophoresis of uncontaminated liquid droplets and gas bubbles in a liquid show quite different behavior from that of rigid particles. The main reason is that flow may also occur within the droplets or bubbles because there is momentum transfer across the interface. The classical notion of a slip plane loses its meaning. Due to the flow inside the droplet or bubble, the tangential velocity of the liquid surrounding the droplet does not have to become zero at the surface of the particle. As a result, the electrophoretic mobility is higher than for a corresponding rigid body. However, to model this situation and to arrive at a conversion of the mobility to a $\zeta$-potential is not a trivial task. As there is no slip plane, even the HS model cannot be applied.

In general, however, droplets or bubbles are not stable without an adsorbed layer of a surface active component (surfactant, polymer, polyelectrolyte, protein). Most layers will make the surface inextensible (rigid) if (nearly) completely covered. In this case, the surface behaves as rigid, and it is possible to use the treatments for rigid particles [3]. Even the presence of the double layer itself, through the primary electroviscous effect, makes momentum transfer to the liquid drop very slight [151].

For partially covered bubbles (as in flotation), the situation is more complex; surface inhomogeneities may occur under the influence of shear, and Marangoni effects become important. Problems arise with regard to the definition of the slip plane, its location, and the charge inhomogeneity [152].

\section{DISCUSSION AND RECOMMENDATIONS}

From the analyses given above, it will be clear that the computation of the $\zeta$-potential from experimental data is not always a trivial task. For each method, the fundamental requirement for the conversion of experimental data is that models should be correctly used within their range of applicability. The bottom line is that the $\zeta$-potential does exist as a characteristic of a charged surface under fixed conditions. This is fortunate, because otherwise, how could two authors compare their results with the same material using two electrokinetic techniques, or even two different versions of the same technique?

This means that we must focus on the correct use of the existing theories, and in their improvement, if necessary. The sometimes-asked question: "Is the computed $\zeta$-potential independent of the technique used?" must be answered with yes, because $\zeta$-potentials are unique characteristics for the charge state of interfaces under given conditions. Whether by different techniques identical $\zeta$-potentials are indeed observed depends on the quality of both the technique and the interpretation. Measuring different electrokinetic properties for a given material in a given liquid medium, and checking the equality of $\zeta$-potentials using appropriate models, is what Lyklema calls an electrokinetic consistency test [3], and what Dukhin and Derjaguin called integrated electrosurface investigation [5].

Another related question that could be asked is "Is the $\zeta$-potential the only property characterizing the electrical equilibrium state of the interfacial region?" The answer is "most often it is not". Most recent models and experimental results demonstrate that in the majority of cases the conductivity behind the slip plane must also be taken into account. This implies that for a correct evaluation, the $D u$ number should also be measured. Using $D u$ only to characterize the interface has, in general, no practical interest either. One experimental electrokinetic technique suffices only if $K^{\sigma \mathrm{i}} / K^{\sigma \mathrm{d}}$ is small because then $D u\left(\zeta, K^{\sigma \mathrm{i}}\right) \approx D u(\zeta, 0)=D u(\zeta)$. The problem now is how does one know that $K^{\sigma \mathrm{i}}$ is in fact that small? We have some possibilities:

- If we have also access to the value of the potentiometrically measured surface charge, $\sigma^{0}$, the values of $\sigma^{\mathrm{ek}}$ (calculated from the value of the $\zeta$-potential obtained by neglecting conductance behind the slip plane) and $\sigma^{0}$ can be compared. This comparison allows us to reach a first estimate 
of $\sigma^{\mathrm{i}}$. From $\sigma^{\mathrm{i}}, K^{\sigma \mathrm{i}}$ could be estimated assuming - as appears to be the case, at least for monovalent counterions - that the mobilities of ions in the inner layer are comparable to those in the bulk. When $D u\left(\zeta, K^{\sigma \mathrm{i}}\right) \approx D u(\zeta, 0)=D u(\zeta)$, the calculated value of $\zeta$ is correct, otherwise, it has to be recalculated taking the inner-layer conduction into account.

- In the case of capillaries or plugs, the total conductivity and the streaming potential can be measured. An initial value of $K^{\sigma \mathrm{d}}$ can be obtained from $\zeta$ using Bikerman's equation, and $K^{\sigma \mathrm{i}}$ can be calculated. The improved value of $\zeta$ can now be obtained using a suitable model including innerlayer conduction, and again $K^{\sigma \mathrm{d}}$ and $K^{\sigma \mathrm{i}}$ can be obtained. If the difference between $\zeta$ computed without and with finite $K^{\sigma \mathrm{i}}$ is high, we should go back to the calculation of $\zeta$ and iterate till the difference between two steps is small.

If these approaches are not possible, there is no other way but employing different electrokinetic techniques on the same sample and performing the consistency test. In this respect, the best procedure would be using one technique in which neglecting $K^{\sigma i}$ underestimates $\zeta$ (electrophoresis, streaming potential, e.g.), and another in which $\zeta$ is overestimated (dc conductivity, dielectric dispersion) [3].

For practical reasons-not every worker has available the numerical routines required for nonanalytical theories—another question emerges: "When can we use with confidence HS equations for the different types of electrokinetic data?"

Although most published data on $\zeta$-potentials are based on the various versions of the HS equation for EKP, let us stress that this approach is correct only if (eq. 2) $\kappa a \gg>1$, where $a$ is a characteristic dimension of the system (curvature radius of the solid particle, capillary radius, equivalent pore radius of the plug,...), and furthermore, the surface conductance of any kind must be low, i.e., $D u\left(\zeta, K^{\sigma i}\right)$ $\ll 1$. Thus, in the absence of independent information about $K^{\sigma \mathrm{i}}$, additional electrokinetic determinations can only be avoided for sufficiently large particles and high electrolyte concentrations.

Another caveat can be given, even if the previously mentioned conditions on dimensions and Dukhin number are met. For concentrated systems, the possibility of the overlap of the double layers of neighboring particles cannot be neglected if the concentration of the dispersed solids in a suspension or a plug is high. In such cases, the validity of the HS equation is also doubtful and cell models for either electrophoresis, streaming potential, or electro-osmosis are required. Use of the latter two kinds of experiments or of electroacoustic or LFDD measurements is recommended. In all cases, a proper model accounting for interparticle interaction must be available.

\section{APPENDIX I. CALCULATION OF THE LOW-FREQUENCY DIELECTRIC DISPERSION OF SUSPENSIONS}

Neglecting SLC, the complex conductivity increment is related to the dipole coefficient as follows [62-64]:

$$
\delta K^{*}(\omega)=\frac{3 \phi}{a^{3}} K_{\mathrm{L}} C_{0}^{*}=\left.\delta K^{*}\right|_{\omega \rightarrow 0}+9 \phi K_{\mathrm{L}} \frac{\left(R^{+}-R^{-}\right) H}{2 A S} \frac{i \omega \tau_{\alpha}}{1+\sqrt{S} \sqrt{i \omega \tau_{\alpha}}+i \omega \tau_{\alpha}}
$$

Its low-frequency value is a real quantity:

$$
\left.\delta K^{*}\right|_{\omega \rightarrow 0}=3 \phi K_{\mathrm{L}}\left(\frac{2 D u(\zeta)-1}{2 D u(\zeta)+2}-\frac{3\left(R^{+}-R^{-}\right) H}{2 B}\right)
$$

The dielectric increment of the suspension can be calculated from $\left.\delta K^{*}\right|_{\omega \rightarrow 0}$ as follows:

$$
\delta \varepsilon_{\mathrm{r}}^{*}(\omega)=-\frac{i}{\omega \varepsilon_{0}}\left(\delta K^{*}-\left.\delta K^{*}\right|_{\omega=0}\right)=\frac{9}{2} \phi \varepsilon_{\mathrm{rs}}^{\prime} \frac{\tau_{\alpha}}{\tau_{\mathrm{el}}} \frac{\left(R^{+}-R^{-}\right) H}{A S} \frac{1}{1+\sqrt{S} \sqrt{i \omega \tau_{\alpha}}+i \omega \tau_{\alpha}}
$$


Here:

$$
R^{ \pm}=4 \frac{\exp \left(\mp \frac{z y^{\mathrm{ek}}}{2}\right)-1}{\kappa a}+6 m^{ \pm}\left[\frac{\exp \left(\mp \frac{z y^{\mathrm{ek}}}{2}\right)-1}{\kappa a} \pm z \frac{z y^{\mathrm{ek}}}{\kappa a}\right]
$$

and

$$
\tau_{\alpha}=\frac{a^{2}}{2 D_{\text {ef }}} \frac{1}{S}
$$

is the value of the relaxation time of the low-frequency dispersion. It is assumed that the dispersion medium is an aqueous solution of an electrolyte of $z-z$ charge type. The definitions of the other quantities appearing in eqs. I.1-5 and the $\zeta$-potential are as follows:

$$
\begin{aligned}
& D_{\text {ef }}=\frac{2 D^{+} D^{-}}{D^{+}+D^{-}} \\
& A=4 D u(\zeta)+4 \\
& B=\left(R^{+}+2\right)\left(R^{-}+2\right)-U^{+}-U^{-}-\left(U^{+} R^{-}+U^{-} R^{+}\right) / 2 \\
& S=\frac{B}{A} \\
& H=\frac{\left(R^{+}-R^{-}\right)\left(1-z^{2} \Delta^{2}\right)-U^{+}+U^{-}+z \Delta\left(U^{+}+U^{-}\right)}{A} \\
& U^{ \pm}=\frac{48 m^{ \pm}}{\kappa a} \ln \left[\cosh \frac{z y_{\zeta}}{4}\right] \\
& \Delta=\frac{D^{-}-D^{+}}{z\left(D^{-}+D^{+}\right)}
\end{aligned}
$$

The factor $1 / S$ is of the order of unity, and comparison of eqs. 58 and I.5 leads to the important conclusion that

$$
\frac{\tau_{\alpha}}{\tau_{\mathrm{MWO}}} \approx(\kappa a)^{2}
$$

This means that for the case of $\kappa a>>1$, the characteristic frequency for the $\alpha$-dispersion is $(\kappa a)^{2}$ times lower than that of the Maxwell-Wagner dispersion.

\section{ACKNOWLEDGMENTS}

Financial assistance from IUPAC is gratefully acknowledged. The Coordinators of the MIEP working party wish to thank all members for their effort in preparing their contributions, suggestions, and remarks. 


\section{REFERENCES}

1. S. S. Dukhin. Adv. Colloid Interface Sci. 61, 17 (1995).

2. R. J. Hunter. Foundations of Colloid Science, Chap. 8, Oxford University Press, Oxford (2001).

3. J. Lyklema. Fundamentals of Interfaces and Colloid Science, Vol. II, Chaps. 3,4, Academic Press, New York (1995).

4. R. J. Hunter. Zeta Potential in Colloid Science, Academic Press, New York (1981).

5. S. S. Dukhin and B. V. Derjaguin. "Electrokinetic phenomena", in Surface and Colloid Science, Vol. 7, Chap. 2, E. Matijević (Ed.), John Wiley, New York (1974).

6. A. V. Delgado (Ed.). Interfacial Electrokinetics and Electrophoresis, Marcel Dekker, New York (2001).

7. Manual of symbols and terminology for physicochemical uantities and units, Appendix II: Definitions, terminology and symbols in colloid and surface chemistry. Part I. Pure Appl. Chem. 31, 577 (1972).

8. I. Mills, T. Cvitaš, K. Homann, N. Kallay, K. Kuchitsu. Quantities, Units and Symbols in Physical Chemistry, $2^{\text {nd }}$ ed., Sect. 2.14, International Union of Pure and Applied Chemistry, Blackwell Science, Oxford (1993).

9. A. D. McNaught and A. Wilkinson. Compendium of Chemical Terminology. IUPAC Recommendations, $2^{\text {nd }}$ ed., International Union of Pure and Applied Chemistry, Blackwell Science, Oxford (1997).

10. J. Lyklema. Pure Appl. Chem. 63, 885 (1995).

11. J. Lyklema, S. Rovillard, J. de Coninck. Langmuir 14, 5659 (1998).

12. J. W. Lorimer. J. Membr. Sci. 14, 275 (1983).

13. C. F. Zukoski and D. A. Saville. J. Colloid Interface Sci. 114, 32 (1986).

14. C. S. Mangelsdorf and L. R. White. J. Chem. Soc., Faraday Trans. 86, 2859 (1990).

15. C. S. Mangelsdorf and L. R. White. J. Chem. Soc., Faraday Trans. 94, 2441 (1998).

16. C. S. Mangelsdorf and L. R. White. J. Chem. Soc., Faraday Trans. 94, 2583 (1998).

17. J. Lyklema and M. Minor. Colloids Surf., A 140, 33 (1998).

18. M. von Smoluchowski. In Handbuch der Electrizität und des Magnetismus (Graetz), Vol. II, p. 366, Barth, Leipzig (1921).

19. J. J. Bikerman. Z. Physik. Chem. A163, 378 (1933).

20. J. J. Bikerman. Kolloid Z. 72, 100 (1935).

21. J. J. Bikerman. Trans. Faraday Soc. 36, 154 (1940).

22. R. W. O'Brien and L. R. White. J. Chem. Soc., Faraday Trans. II 74, 1607 (1978).

23. R. W. O'Brien and R. J. Hunter. Can. J. Chem. 59, 1878 (1981).

24. D. C. Henry. Proc. R. Soc. London A133, 106 (1931).

25. H. Ohshima. J. Colloid Interface Sci. 168, 269 (1994).

26. R. P. Tison. J. Colloid Interface Sci. 60, 519 (1977).

27. A. Homola and A. A. Robertson. J. Colloid Interface Sci. 51, 202 (1975).

28. K. Furusawa, Y. Kimura, T. Tagawa. In Polymer Adsorption and Dispersion Stability, E. D. Goddard and B. Vincent (Eds.), ACS Symposium Series Vol. 240, p. 131, American Chemical Society, Washington, DC (1984).

29. M. Minor, A. J. van der Linde, H. P. van Leeuwen, J. Lyklema. J. Colloid Interface Sci. 189, 370 (1997).

30. E. E. Uzgiris. Rev. Sci. Instrum. 45, 74 (1974).

31. R. Ware and W. H. Flygare. J. Colloid Interface Sci. 39, 670 (1972).

32. E. E. Uzgiris. Optics Comm. 6, 55 (1972).

33. E. Malher, D. Martin, C. Duvivier. Studia Biophys. 90, 33 (1982).

34. E. Malher, D. Martin, C. Duvivier, B. Volochine, J. F. Stolz. Biorheology 19, 647 (1982). 
35. K. Oka and K. Furusawa. In Electrical Phenomena at Interfaces, H. Ohshima and K. Furusawa (Eds.), Chap. 8, Marcel Dekker, New York (1998).

36. J. F. Miller, K. Schätzel, B. Vincent. J. Colloid Interface Sci. 143, 532 (1991).

37. F. Manerwatson, W. Tscharnuter, J. Miller. Colloids Surfaces A 140, 53 (1988).

38. J. F. Miller, O. Velev, S. C. C. Wu, H. J. Ploehn. J. Colloid Interface Sci. 174, 490 (1995).

39. Japanese Surface and Colloid Chemical Group (Japan Oil Chemists Soc.). Yukagaku 25, 239 (1975).

40. F. Fairbrother and H. Mastin. J. Chem. Soc. 2319 (1924).

41. D. R. Briggs. J. Phys. Chem. 32, 641 (1928).

42. M. Chang and A. A. Robertson. Can. J. Chem. Eng. 45, 66 (1967).

43. G. J. Biefer and S. G. Mason. Trans. Faraday Soc. 55, 1234 (1959).

44. J. Happel and P. A. Ast. Chem. Eng. Sci. 11, 286 (1960).

45. J. Schurz and G. Erk. Progr. Colloid Polym. Sci. 71, 44 (1985).

46. S. Levine, G. Neale, N. Epstein. J. Colloid Interface Sci. 57, 424 (1976).

47. J. L. Anderson and W. H. Koh. J. Colloid Interface Sci. 59, 149 (1977).

48. S. Levine, J. R. Marriot, G. Neale, N. Epstein. J. Colloid Interface Sci. 52, 136 (1975).

49. D. Burgreen and F. R. Nakache. J. Phys. Chem. 68, 1084 (1964).

50. C. L. Rice and P. Whitehead. J. Phys. Chem. 69, 4017 (1965).

51. R. W. O’Brien and W. T. Perrins. J. Colloid Interface Sci. 99, 20 (1984).

52. D. Erickson and D. Li. J. Colloid Interface Sci. 237, 283 (2001).

53. A. Szymczyk, B. Aoubiza, P. Fievet, J. Pagetti. J. Colloid Interface Sci. 192, 105 (2001).

54. N. Spanos and P. G. Koutsoukos. J. Colloid Interface Sci. 214, 85 (1999).

55. J. M. Gierbers, J. M. Kleijn, M. A. Cohen-Stuart. J. Colloid Interface Sci. 248, 88 (2002).

56. O. El-Gholabzouri, M. A. Cabrerizo, R. Hidalgo-Álvarez. J. Colloid Interface Sci. 261, 386 (2003).

57. A. Pettersson and J. B. Rosenholm. Langmuir 18, 8447 (2002).

58. J. Th. G. Overbeek. In Colloid Science, Vol. I, H. R. Kruyt (Ed.), Chap. V, Elsevier, Amsterdam (1952).

59. C. Werner, R. Zimmermann, T. Kratzmüller. Colloids Surf., A 192, 205 (2001).

60. J. Kijlstra, H. P. van Leeuwen, J. Lyklema. Langmuir 9, 1625 (1993).

61. J. Kijlstra, H. P. van Leeuwen, J. Lyklema. J. Chem. Soc., Faraday Trans. 88, 3441 (1992).

62. C. T. O'Konski. J. Phys. Chem. 64, 605 (1960).

63. S. S. Dukhin and V. N. Shilov. Dielectric Phenomena and the Double Layer in Disperse Systems and Polyelectrolytes, John Wiley, Jerusalem (1974).

64. C. Grosse and V. N. Shilov. J. Phys. Chem. 100, 1771 (1996).

65. B. Nettleblad and G. A. Niklasson. J. Colloid Interface Sci. 181, 165 (1996).

66. E. H. B. DeLacey and L. R. White. J. Chem. Soc. Faraday Trans. II 77, 2007 (1981).

67. V. N. Shilov, A. V. Delgado, F. González-Caballero, C. Grosse. Colloids Surf., A 192, 253 (2001).

68. L. A. Rosen, J. C. Baygents, D. A. Saville. J. Chem. Phys. 98, 4183 (1993).

69. F. J. Arroyo, F. Carrique, T. Bellini, A. V. Delgado. J. Colloid Interface Sci. 210, 194 (1999).

70. E. Vogel and H. Pauli. J. Chem. Phys. 89, 3830 (1988).

71. Yu. B. Borkovskaja and V. N. Shilov. Colloid J. 54, 173 (1992).

72. V. N. Shilov and Yu. B. Borkovskaja. Colloid J. 56, 647 (1994).

73. A. V. Delgado, F. J. Arroyo, F. Gonzalez-Caballero, V. N. Shilov, Y. Borkovskaja. Colloids Surf., A 140, 139 (1998).

74. F. Carrique, F. J. Arroyo, M. L. Jiménez, A. V. Delgado. J. Chem. Phys. 118, 1945 (2003).

75. H. Fricke and H. J. Curtis. J. Phys. Chem. 41, 729 (1937).

76. F. J. Arroyo, F. Carrique, A. V. Delgado. J. Colloid Interface Sci. 217, 411 (1999).

77. M. M. Springer, A. Korteweg, J. Lyklema. J. Electroanal. Chem. 153, 55 (1983).

78. K. Lim and E. I. Frances. J. Colloid Interface Sci. 110, 201 (1986). 
79. C. Grosse, A. J. Hill, K. R. Foster. J. Colloid Interface Sci. 127, 167 (1989).

80. C. Grosse and M. C. Tirado. Mater. Res. Soc. Symp. Proc. 430, 287 (1996).

81. M. L. Jiménez, F. J. Arroyo, J. van Turnhout, A. V. Delgado. J. Colloid Interface Sci. 249, 327 (2002).

82. R. W O'Brien. J. Fluid Mech. 212, 81 (1990).

83. R. W. O’Brien, B. R. Midmore, A. Lamb, R. J. Hunter. Faraday Discuss. Chem Soc. 90, 301 (1990).

84. R. W. O’Brien. J. Fluid Mech. 190, 71 (1988).

85. R. W. O’Brien, D. Cannon, W. N. Rowlands. J. Colloid Interface Sci. 173, 406 (1995).

86. R. J. Hunter. Colloids Surf., A 141, 37 (1998).

87. R. J. Hunter and R. W. O’Brien. Colloids Surf., A 126, 123 (1997).

88. R. W. O’Brien. U.S. Patent 5,059,909 (1991).

89. R. P. Sawatsky and A. J. Babchin. J. Fluid Mech. 246, 321 (1993).

90. C. S. Mangelsdorf and L. R. White. J. Colloid Interface Sci. 160, 275 (1993).

91. C. S. Mangelsdorf and L. R. White. J. Chem. Soc., Faraday Trans. 88, 3567 (1992).

92. S. E. Gibb and R. J. Hunter. J. Colloid Interface Sci. 224, 99 (2000).

93. M. Loewenberg and R. W. O’Brien. J. Colloid Interface Sci. 150, 158 (1992).

94. M. Loewenberg. J. Fluid Mech. 278, 149 (1994).

95. H. Ohshima. J. Colloid Interface Sci. 185, 131 (1997).

96. B. J. Marlow, D. Fairhurst, H. P. Pendse. Langmuir 4, 611 (1988).

97. S. Levine and G. Neale. J. Colloid Interface Sci. 47, 520 (1974).

98. S. Levine and G. Neale. J. Colloid Interface Sci. 49, (1974).

99. R. W. O’Brien, W. N. Rowlands, R. J. Hunter. In Electro-acoustics for Characterization of Particulates and Suspensions, S. B. Malghan (Ed.), NIST Special Publication 856, pp. 1-22, National Institute of Standards and Technology, Washington, DC (1993).

100. R. J. Hunter. "Electrokinetic characterization of emulsions", in Encyclopaedic Handbook of Emulsion Technology, J. Sjoblom (Ed.), Chap. 7, Marcel Dekker, New York (2001).

101. P. Rider and R. W. O'Brien. J. Fluid Mech. 257, 607 (1993).

102. J. Ennis, A. A. Shugai, S. L. Carnie. J. Colloid Interface Sci. 223, 21 (2000).

103. S. B. Johnson, A. S. Russell, P. J. Scales. Colloids Surf., A 141, 119 (1998).

104. R. W. O’Brien, A. Jones, W. N. Rowlands. Colloids Surf., A 218, 89 (2003).

105. A. S. Dukhin, V. N. Shilov, H. Ohshima, P. J. Goetz. Langmuir 15, 6692 (1999).

106. A. H. Harker and J. A. G. Temple. J. Phys. D.: Appl. Phys. 21, 1576 (1988).

107. R. L. Gibson and M. N. Toksoz. J. Acoust. Soc. Amer. 85, 1925 (1989).

108. A. S. Dukhin and P. J. Goetz. Langmuir 12, 4987 (1996).

109. A. S. Ahuja. J. Appl. Phys. 44, 4863 (1973).

110. S. Kuwabara. J. Phys. Soc. Jpn. 14, 527 (1959).

111. V. N. Shilov, N. I. Zharkikh, Y. B. Borkovskaya. Colloid J. 43, 434 (1981).

112. A. S. Dukhin, V. N. Shilov, H. Ohshima, P. J. Goetz. In Interfacial Electrokinetics and Electrophoresis, A. V. Delgado (Ed.), Chap. 17, Marcel Dekker, New York (2001).

113. R. J. Hunter and R. W. O'Brien. In Encyclopedia of Colloid and Surface Science, A. Hubbard (Ed.), p. 1722, Marcel Dekker, New York (2002).

114. H. Ohshima. In Electrical Phenomena at Interfaces. Fundamentals, Measurements and Applications, H. Ohshima and K. Furusawa (Eds.), Chap. 2, Marcel Dekker, New York (2002).

115. F. Carrique, F. J. Arroyo, A. V. Delgado. J. Colloid Interface Sci. 206, 206 (1998).

116. S. S. Dukhin. Adv. Colloid Interface Sci. 44, 1 (1993).

117. R. W. O'Brien and D. N. Ward. J. Colloid Interface Sci. 121, 402 (1988).

118. M. C. Fair and J. L. Anderson. J. Colloid Interface Sci. 127, 388 (1989).

119. D. Velegol, J. L. Anderson, Y. Solomentsev. In Interfacial Electrokinetics and Electrophoresis, A. V. Delgado (Ed.), Chap. 6, Marcel Dekker, New York (2002). 
120. J. Y. Kim and B. J. Yoon. In Interfacial Electrokinetics and Electrophoresis, A. V. Delgado (Ed.), Chap. 7, Marcel Dekker, New York (2002).

121. C. Grosse and V. N. Shilov. J. Colloid Interface Sci. 193, 178 (1997).

122. C. Grosse, S. Pedrosa, V. N. Shilov. J. Colloid Interface Sci. 220, 31 (1999).

123. S. S. Dukhin, R. Zimmermann, C. Werner. Colloids Surf., A 195, 103 (2001).

124. J. Y. Walz. Adv. Colloid Interface Sci. 74, 119 (1998).

125. S. A. Allison. Macromolecules 29, 7391 (1996).

126. M. Teubner. J. Chem. Phys. 76, 5564 (1982).

127. M. C. Fair and J. L. Anderson. Langmuir 8, 2850 (1992).

128. D. Velegol, J. L. Anderson, S. Garoff. Langmuir 12, 675 (1996).

129. J. L. Anderson, D. Velegol, S. Garoff. Langmuir 16, 3372 (2000).

130. J. L. Anderson. J. Colloid Interface Sci. 105, 45 (1985).

131. H. van Olphen. An Introduction to Clay Colloid Chemistry, John Wiley, New York (1977).

132. S. Akari, W. Schrepp, D. Horn. Langmuir 12, 857 (1996).

133. K. E. Bremmell, G. J. Jameson, S. Biggs. Colloids Surf., A 139, 199 (1998).

134. M. A. Cohen-Stuart, F. H. W. H. Waajen, S. S. Dukhin. Colloid Polym. Sci. 262, 423 (1984).

135. L. K. Koopal, V. Hlady, J. Lyklema. J. Colloid Interface Sci. 121, 49 (1988).

136. L. Kong, J. K. Beattie, R. J. Hunter. Phys. Chem. Chem. Phys. 3, 87 (2001).

137. H. Ohshima. J. Colloid Interface Sci. 163, 474 (1995).

138. H. Ohshima and K. Makino. Colloids Surf., A 109, 71 (1996).

139. H. N. Stein. In Interfacial Electrokinetics and Electrophoresis, A. V. Delgado (Ed.), Chap. 21, Marcel Dekker, New York (2002).

140. R. Hidalgo-Álvarez, A. Martín, A. Fernández, D. Bastos, F. Martínez, F. J. de las Nieves. Adv. Colloid Interface Sci. 67, 1 (1996).

141. H. Ohshima. In Interfacial Electrokinetics and Electrophoresis, A. V. Delgado, (Ed.), Chap. 5, Marcel Dekker, New York (2002).

142. H. J. Keh. In Interfacial Electrokinetics and Electrophoresis, A. V. Delgado (Ed.), Chap. 15, Marcel Dekker, New York (2002).

143. C. Tanford and J. A. Reynolds. Biochim. Biophys. Acta 457, 133 (1976).

144. D. Stigter. Cell Biophys. 11, 139 (1987).

145. D. Stigter. J. Phys. Chem. B 104, 3402 (2000).

146. R. J. Hill, D. A. Saville, W. B. Russel. J. Colloid Interface Sci. 258, 56 (2003).

147. A. Martín-Rodríguez, J. L. Ortega-Vinuesa, R. Hidalgo-Álvarez. In Interfacial Electrokinetics and Electrophoresis, A. V. Delgado (Ed.), Chap. 22, Marcel Dekker, New York (2002).

148. E. Lee, F. Y. Yen, J. P. Hsu. In Interfacial Electrokinetics and Electrophoresis, A. V. Delgado (Ed.), Chap. 23, Marcel Dekker, New York (2002).

149. R. Barchini, H. P. van Leewen, J. Lyklema. Langmuir 16, 8238 (2000).

150. A. Van der Wal, M. Minor, W. Norde, A. J. B. Zehnder, J. Lyklema. Langmuir 13, 165 (1997).

151. S. A. Nespolo, M. A. Bevan, D. Y. C. Chan, F. Grieser, G. W. Stevens. Langmuir 17, 7210 (2001).

152. C. Yang, T. Dabros, D. Li, J. Czarnecki, J. H. Masliyah. J. Colloid Interface Sci. 243, 128 (2001). 


\section{LIST OF SYMBOLS}

Note: SI base (or derived) units are given in parentheses for all quantities, except dimensionless ones.

$a(\mathrm{~m})$

$A_{\mathrm{c}}\left(\mathrm{m}^{2}\right)$

$A_{\mathrm{c}}^{\mathrm{ap}}\left(\mathrm{m}^{2}\right)$

$A_{\mathrm{ESA}}(\mathrm{Pa})$

$b(\mathrm{~m})$

$c\left(\mathrm{~mol} \mathrm{~m}^{-3}\right)$

$c_{+}\left(c_{-}\right)\left(\mathrm{mol} \mathrm{m}^{-3}\right)$

$C_{0}^{*}$

$d(\mathrm{~m})$

$d^{*}(\mathrm{C} \mathrm{m})$

$d^{\mathrm{ek}}(\mathrm{m})$

$D\left(\mathrm{~m}^{2} \mathrm{~s}^{-1}\right)$

$D_{+}\left(D_{-}\right)\left(\mathrm{m}^{2} \mathrm{~s}^{-1}\right)$

Du

$D u^{\mathrm{d}}$

$D u^{\mathrm{i}}$

$e(\mathrm{C})$

$\boldsymbol{E}\left(\mathrm{V} \mathrm{m}^{-1}\right)$

$E_{\text {sed }}\left(\mathrm{V} \mathrm{m}^{-1}\right)$

$E_{\mathrm{t}}\left(\mathrm{V} \mathrm{m}^{-1}\right)$

$F\left(\mathrm{C} \mathrm{mol}^{-1}\right)$

$f_{1}(\kappa a), F_{1}\left(\kappa a, K_{\mathrm{p}}\right)$

$I(\mathrm{~A})$

$I_{0}, I_{1}$

$I_{\mathrm{c}}(\mathrm{A})$

$I_{\mathrm{CV}}(\mathrm{A})$

$I_{\text {str }}(\mathrm{A})$

$j^{\sigma}\left(\mathrm{A} \mathrm{m}^{-1}\right)$

$j_{\text {str }}\left(\mathrm{A} \mathrm{m}^{-2}\right)$

$k\left(\mathrm{~J} \mathrm{~K}^{-1}\right)$

$K\left(\mathrm{~S} \mathrm{~m}^{-1}\right)$

$K_{\mathrm{DC}}\left(\mathrm{S} \mathrm{m}^{-1}\right)$

$K_{\mathrm{L}}\left(\mathrm{S} \mathrm{m}^{-1}\right)$

$K_{\mathrm{L}}^{\infty}\left(\mathrm{S} \mathrm{m}^{-1}\right)$

$K_{\mathrm{p}}\left(\mathrm{S} \mathrm{m}^{-1}\right)$

$K_{\text {plug }}\left(\mathrm{S} \mathrm{m}^{-1}\right)$

$K_{\text {pef }}\left(\mathrm{S} \mathrm{m}^{-1}\right)$

$K_{\text {rel }}$

$K^{*}\left(\mathrm{~S} \mathrm{~m}^{-1}\right)$

$K^{\sigma}(\mathrm{S})$

$K^{\sigma \mathrm{d}}(\mathrm{S})$

$K^{\sigma \mathrm{\sigma i}}(\mathrm{S})$

$L(\mathrm{~m})$

$L^{\text {ap }}(\mathrm{m})$

$L_{D}(\mathrm{~m})$

$m$ particle radius, local curvature radius, capillary radius

capillary cross-section

apparent (externally measured) capillary cross-section

electrokinetic sonic amplitude

half distance between neighboring particles

electrolyte concentration

concentration of cations (anions)

dipole coefficient of particles

distance between the surface and the outer Helmholtz plane

complex dipole moment

distance between the surface and the slip plane

diffusion coefficient of counterions (or average diffusion coefficient of ions)

diffusion coefficient of cations (anions)

Dukhin number

Dukhin number associated with diffuse-layer conductivity

Dukhin number associated with stagnant-layer conductivity

elementary charge

applied electric field

sedimentation field

tangential component of external field

Faraday constant

Henry's functions

electric current intensity

zeroth- (first-) order modified Bessel functions of the first kind

conduction current

colloid vibration current

streaming current

surface current density

streaming current density

Boltzmann constant

total conductivity of a colloidal system

direct current conductivity of a suspension

conductivity of dispersion medium

conductivity of a highly concentrated ionic solution

conductivity of particles

conductivity of a plug of particles

effective conductivity of particles

ratio between particle and liquid conductivities

complex conductivity of a suspension

surface conductivity

diffuse-layer surface conductivity

stagnant-layer surface conductivity

capillary length, characteristic dimension

apparent (externally measured) capillary cross-section

ionic diffusion length

dimensionless ionic mobility of counterions 


$m_{+}\left(m_{-}\right)$
$n\left(\mathrm{~m}^{-3}\right)$
$N_{\mathrm{A}}\left(\mathrm{mol}^{-1}\right)$
$n_{i}\left(\mathrm{~m}^{-3}\right)$
$Q_{\mathrm{eo}}\left(\mathrm{m}^{3} \mathrm{~s}^{-1}\right)$
$Q_{\mathrm{eo}, \mathrm{E}}\left(\mathrm{m}^{4} \mathrm{~s}^{-1} \mathrm{~V}^{-1}\right)$
$Q_{\mathrm{eo}, \mathrm{I}}\left(\mathrm{m}^{3} \mathrm{C}^{-1}\right)$
$r(\mathrm{~m})$
$R(\mathrm{~m})$
$R_{\mathrm{s}}(\Omega)$
$R_{\infty}(\Omega)$
$T(\mathrm{~K})$
$u_{\mathrm{d}}^{*}\left(\mathrm{~m}^{2} \mathrm{~s}^{-1} \mathrm{~V}^{-1}\right)$
$U_{\mathrm{CV}}(\mathrm{V})$
$u_{\mathrm{e}}\left(\mathrm{m}^{2} \mathrm{~s}^{-1} \mathrm{~V}^{-1}\right)$
$U_{\mathrm{sed}}(\mathrm{V})$
$U_{\mathrm{str}}(\mathrm{V})$
$v_{\mathrm{e}}\left(\mathrm{m} \mathrm{s}^{-1}\right)$
$v_{\mathrm{eo}}\left(\mathrm{m} \mathrm{s}^{-1}\right)$
$v_{\mathrm{L}}\left(\mathrm{m} \mathrm{s}^{-1}\right)$
$y^{\mathrm{ek}}$
$z$
$z_{i}$
$\alpha$

$\beta(\mathrm{m})$

$\Gamma_{i}\left(\mathrm{~m}^{-2}\right)$

$\delta c\left(\mathrm{~mol} \mathrm{~m}^{-3}\right)$

$\delta K^{*}\left(\mathrm{~S} \mathrm{~m}^{-1}\right)$

$\delta \varepsilon_{\mathrm{r}}^{*}$

$\Delta p(\mathrm{~Pa})$

$\Delta p_{\text {eo }}(\mathrm{Pa})$

$\Delta V_{\text {ext }}(\mathrm{V})$

$\Delta \varepsilon(0)$

$\Delta \varepsilon_{\mathrm{d}}(0)$

$\Delta \rho\left(\mathrm{kg} \mathrm{m}^{-3}\right)$

$\varepsilon^{*}\left(\mathrm{~F} \mathrm{~m}^{-1}\right)$

$\varepsilon_{\mathrm{r}}^{*}$

$\varepsilon_{\mathrm{rp}}$

$\varepsilon_{\mathrm{rp}}^{*}$

$\varepsilon_{\mathrm{rs}}$

$\varepsilon_{\mathrm{rs}}^{*}$

$\varepsilon_{0}\left(\mathrm{~F} \mathrm{~m}^{-1}\right)$

$\zeta(\mathrm{V})$

$\zeta_{\text {app }}(\mathrm{V})$

$\eta$ (Pa s)

$\kappa\left(\mathrm{m}^{-1}\right)$

$\rho\left(\mathrm{kg} \mathrm{m}^{-3}\right)$ dimensionless ionic mobility of cations (anions)

number concentration of particles

Avogadro constant

number concentration of type $i$ ions

electro-osmotic flow rate

electro-osmotic flow rate per electric field

electro-osmotic flow rate per current

spherical or cylindrical radial coordinate

roughness of a surface

electrical resistance of a capillary or porous plug in an arbitrary solution

electrical resistance of a capillary or porous plug in a concentrated ionic solution

thermodynamic temperature

dynamic electrophoretic mobility

colloid vibration potential

electrophoretic mobility

sedimentation potential

streaming potential

electrophoretic velocity

electro-osmotic velocity

liquid velocity in electrophoresis cell

dimensionless $\zeta$-potential

common charge number of ions in a symmetrical electrolyte

charge number of type $i$ ions

relaxation of double-layer polarization, degree of electrolyte dissociation, dimensionless parameter used in electroacoustics

distance between the solid surface and the inner Hemholtz plane (see also eq. 45

for another use of this symbol)

surface concentration of type $i$ ions

field-induced perturbation of electrolyte amount concentration

conductivity increment of a suspension

relative dielectric increment of a suspension

applied pressure difference

electro-osmotic counter-pressure

applied potential difference

low-frequency dielectric increment per volume fraction

value of $\Delta \varepsilon(0)$ for suspensions with low volume fractions

density difference between particles and dispersion medium

complex electric permittivity of a suspension

complex relative permittivity of a suspension

relative permittivity of the particle

complex relative permittivity of a particle

relative permittivity of the dispersion medium

complex relative permittivity of the dispersion medium

electric permittivity of vacuum

electrokinetic or $\zeta$-potential

electrokinetic or $\zeta$-potential not corrected for the effect of particle concentration dynamic viscosity

reciprocal Debye length

density of dispersion medium 


$\begin{array}{ll}\rho_{\mathrm{p}}\left(\mathrm{kg} \mathrm{m}^{-3}\right) & \text { density of particles } \\ \rho_{\mathrm{s}}\left(\mathrm{kg} \mathrm{m}^{-3}\right) & \text { density of a suspension } \\ \sigma^{\mathrm{d}}\left(\mathrm{C} \mathrm{m}^{-2}\right) & \text { diffuse charge density } \\ \sigma^{\mathrm{ek}}\left(\mathrm{C} \mathrm{m}^{-2}\right) & \text { electrokinetic charge density } \\ \sigma^{\mathrm{i}}\left(\mathrm{C} \mathrm{m}^{-2}\right) & \text { surface charge density at the inner Helmholtz plane } \\ \sigma^{0}\left(\mathrm{C} \mathrm{m}^{-2}\right) & \text { titratable surface charge density } \\ \tau_{\mathrm{MWO}}(\mathrm{s}) & \text { characteristic time of the Maxwell-Wagner-O'Konski relaxation } \\ \tau_{\alpha}(\mathrm{s}) & \text { relaxation time of the low-frequency dispersion } \\ \phi & \text { volume fraction of solids } \\ \phi_{\mathrm{L}} & \text { volume fraction of liquid in a plug } \\ \psi^{\mathrm{d}}(\mathrm{V}) & \text { diffuse-layer potential } \\ \psi^{\mathrm{i}}(\mathrm{V}) & \text { inner Helmholtz plane potential } \\ \psi^{0}(\mathrm{~V}) & \text { surface potential } \\ \omega^{-1}\left(\mathrm{~s}^{-1}\right) & \text { angular frequency of an ac electric field } \\ \omega_{\mathrm{MWO}}\left(\mathrm{s}^{-1}\right) & \text { Maxwell-Wagner-O'Konski characteristic frequency } \\ \omega_{\alpha}\left(\mathrm{s}^{-1}\right) & \text { characteristic frequency of the } \alpha \text {-relaxation } \\ \omega_{\alpha \mathrm{d}}\left(\mathrm{s}^{-1}\right) & \text { characteristic frequency of the } \alpha \text {-relaxation for a dilute suspension }\end{array}$

